\title{
STABILITY IN HIGH DIMENSIONAL STEEP REPELLING POTENTIALS.
}

\author{
A. RAPOPORT, V. ROM-KEDAR AND D. TURAEV
}

\begin{abstract}
The appearance of elliptic periodic orbits in families of $n$-dimensional smooth repelling billiard-like potentials that are arbitrarily steep is established for any finite $n$. Furthermore, the stability regions in the parameter space scale as a power-law in $1 / n$ and in the steepness parameter. Thus, it is shown that even though these systems have a uniformly hyperbolic (albeit singular) limit, the ergodicity properties of this limit system are destroyed in the more realistic smooth setting. The considered example is highly symmetric and is not directly linked to the smooth many particle problem. Nonetheless, the possibility of explicitly constructing stable motion in smooth $n$ degrees of freedom systems limiting to strictly dispersing billiards is now established.
\end{abstract}

\section{INTRODUCTION}

At sufficiently high temperature, many-particle gas systems show fast decay of correlation, and, for most initial configurations, the time averages of this system and the appropriately defined ensemble averages coincide. This fundamental observation of Boltzmann lead to the development of the theory of statistical mechanics. It was further suggested by Boltzmann that at such temperatures the particles interaction resembles that of hard spheres, independent of the details of their effective potentials, hence, that a gas of hard spheres supplies an instructive universal model for studying statistical properties of gases. Notably, Boltzmann considered the many-particle case. Krylov explained that the fast decay of correlations of the hard sphere model is caused by the instability associated with the dispersive nature of the collision between the hard spheres, similar to the instabilities that appear in geodesic flows with negative curvature [15]. Sinai found that this instability appears in any dispersing billiard ${ }^{1}$ geometry (later on called Sinai billiards ${ }^{2}$ ) in any dimension, and set the mathematical foundation for rigorously studying such systems. Sinai proved, in his seminal works $[30,31]$, that such systems are ergodic and hyperbolic in the two dimensional billiard case. He further stated (the Sinai-Boltzmann conjecture ${ }^{3}$ ) that if one considers the motion of $N$ hard spheres on a $d$-dimensional torus (an $n=N d$ dimensional system), this motion will be mixing ${ }^{4}$ for any $d \geq 2$ and $N \geq 2$. In particular, the SinaiBoltzmann conjecture means that for any $N, d \geq 2$, ergodicity is achieved independently of the number of particles because of the universal nature of the instability associated with the convex particles collision.

\footnotetext{
${ }^{1}$ The behavior of a point particle traveling with a constant speed in a region $D$, undergoing elastic collisions at the region's boundary, is known as the billiard problem. The billiard is dispersing if its boundary is piecewise strictly concave when looking from the billiards's interior.

${ }^{2}$ Strictly dispersing billiards for which the smooth boundary components intersect at positive angles (no cusps are allowed).

${ }^{3}$ Proved initially for the $N=d=2$ by Sinai[31], then for the $N=2, d=3$ by [32] whereas the proofs for the most general higher dimensional cases appeared only recently [11, 12, 13, 14, 26, 25, 29, 27, 28]

4 on the reduced manifold, eliminating the total energy and momenta conservation laws.
} 
We propose that the study of real particles, with smooth potentials, or, more generally, in studying Hamiltonians with smooth steep $n$-dimensional potentials, may shed light on the role of dimensionality in this problem. Thus, to formalize this notion, we consider a Hamiltonian

$$
H=\sum_{i=1}^{n} \frac{p_{i}^{2}}{2}+W(x ; \varepsilon)
$$

where $W(x ; \varepsilon)$ is a smooth potential that becomes a hard-wall potential ${ }^{5}$ in the limit $\varepsilon \rightarrow 0$ :

$$
W(x ; \varepsilon) \underset{\varepsilon \rightarrow 0}{\rightarrow} \begin{cases}0 & x \in D \backslash \partial D \\ c & x \in \partial D .\end{cases}
$$

In general, studying (1.1) for a finite $\varepsilon$ value is a formidable task. Boltzmann's insight and Sinai's theory, in which the $n$-dimensional nonlinear system is replaced by the study of billiards, serve as a great simplification. To mimic the soft nature of the particles interactions and still obtain a tractable system, finite-range axis-symmetric potentials were introduced. It was established that these systems may be studied by a modified (non-smooth) billiard map, and thus that in two dimensions some configurations remain ergodic [30, 16, 6, 3], while other configurations may possess stability islands [2, 5]. More recently, some higher dimensional configurations were proved to be hyperbolic [4].

Yet, it was noticed in [34] that the behavior of any smooth approximation has to be fundamentally different from the discontinuous behavior of the billiards. Indeed, in mathematical terms the Krylov-Sinai instability translates to the existence of a universal hyperbolic structure in any dispersing billiard problem. More precisely, the family of cones $d x \cdot d p>0$ is forward invariant with respect to the billiard flow in the dispersing case independent of the details of the billiard's shape. After each reflection from the billiard's boundary, the cones are mapped into each other with flipped orientation (the normal component of the momentum $p$ changes sign, while all other components are preserved), see $[31,36,34]$. In particular, nearby orbits experiencing different number of reflections (i.e. near tangencies or near corners), have unstable manifolds with opposite orientability properties - one orientable and the other non-orientable [34]. Such a discontinuous dependence of the unstable manifold on initial conditions in smooth uniformly hyperbolic systems is impossible.

On the other hand, the hyperbolic structure near regular orbits of the billiard (e.g. periodic orbits which are bounded away from the singularity set) is typically inherited by steep billiard-like potentials [34, 21]. It follows that the Krylov-Sinai instability mechanism indeed controls the smooth dynamics but only for some limited time scale, after which the non-hyperbolic behavior which stems from the billiard singularities will prevail. Therefore, we propose that the dependence of this instability time scale on the number of particles and other parameters is the most relevant question in the study of many-particle systems.

One concludes then, that in order to study the dynamics of real particles, one needs to study (1.1) for smooth steep potentials, utilizing the theoretical advancements regarding the singular billiard limit as a tool in this study. This approach requires a well-defined limiting procedure that is well developed by now [17, 22, 21].

This formulation was first introduced in the most general two-dimensional setting of Sinai billiards (not necessarily axis-symmetric, nor of finite range) in [22]. After proving

\footnotetext{
5 here $c>0$ may be finite or infinite, and we always take the particle's energy, $h$, to be positive and strictly smaller than $c$ so that the particle cannot cross $\partial D$.
} 
that regular hyperbolic orbits of the billiard persist in the smooth flow, two mechanisms by which the billiards ergodicity property is destroyed were identified $[22,35]$. One such mechanism is a tangency: periodic orbits or homoclinic orbits which are tangent to the billiard's boundary produce islands of stability [22]. Another mechanism are corners - a sequence of regular reflections which begins and ends in a corner (termed a corner polygon) may, under some prescribed conditions, produce stable periodic orbits [35]. In both cases it was shown that a two-parameter family of potentials $W(x ; \mu, \varepsilon)(\varepsilon$ is the steepness parameter and $\mu$ is responsible for a regular continuous change of the billiard's geometry) possesses a wedge in the $(\mu, \varepsilon)$-plane, at which the Hamiltonian flow has an elliptic periodic orbit. This orbit limits to the tangent billiard orbit/ the corner polygon as $\varepsilon \rightarrow 0$. These findings were shown to correctly describe the motion of cold atoms in atom-optics billiards in laboratory experiments [10].

What would one expect in the multi-dimensional case? Can there be other type of universal instabilities, besides the Krylov-Sinai one, which makes such systems ergodic for sufficiently steep potentials? Namely, would the billiard's ergodicity be preserved for $n$ dimensional steep billiard-like potentials when $n \geq 3$ ? While there are some conjectures regarding the generic appearance of islands in smooth $n$ degrees of freedom systems, results of this nature appeared only in the case of $C^{1}$-flows and assume the systems are not partially hyperbolic (see $[33,19,1,24]$ ), which is the heart of the problem here. In fact, the above described mechanism of orientation flipping, which corresponds to a direct generalization of our previous two-dimensional results (e.g. [22]) to dispersing $n$-dimensional billiards, will produce orbits which have one pair of imaginary multipliers (ruining hyperbolicity), yet all the other $n-2$ pairs can still correspond to hyperbolic behavior. Namely, such a direct generalization will ruin hyperbolicity yet it is not necessarily going to ruin ergodicity in the smooth case, as the existence of some uniform partially hyperbolic structure is not ruled out by this mechanism. This intuition might lead one to believe that the mechanisms described in $[22,35]$ for ruining ergodicity are inherently two-dimensional.

Recently, it was demonstrated numerically that regions of effective stability, hereafter called islands, are created in steep dispersing three-dimensional billiards for what appears to be arbitrarily small $\varepsilon$ [20]. Before further describing this construction and its current generalization to the $n$ degrees of freedom case, let us discuss the issue of islands in the multi-dimensional context.

As opposed to the two-dimensional situation, due to the possible existence of Arnold diffusion, one cannot claim that in the vicinity of non-degenerate non-resonant elliptic orbit there exists an invariant open neighborhood (on energy surfaces or on the full phase space). Nonetheless, by KAM theory, near such elliptic orbits there exists a positive measure set foliated by KAM-tori that corresponds to trajectories which remain forever near the elliptic trajectory. Furthermore, while other trajectories in this neighborhood may perhaps escape, this can take exponentially long time $[18,7]$ (namely, such islands may correspond to highdimensional dynamical traps, generalizing the $2 \mathrm{~d}$ stickiness phenomena). Thus, hereafter, an island in the multi-dimensional context will be defined as the small neighborhood of the elliptic orbit which is effectively stable [7], bearing in mind that only in the two degrees of freedom case this neighborhood is known to correspond to an invariant set.

The islands constructed in [20] are produced by a highly symmetric orbit of the smooth system which visits the vicinity of a symmetric 3 -corner. The 3-corner is a point at which three smooth spheres of identical radius intersect in a symmetric fashion, so that only one characteristic parameter $\mu$ controls the angle of their intersection $(\mu=0$ corresponds to a cusp whereas $\mu=1$ corresponds to a complete overlap of the spheres, see Figure 
1). It is demonstrated numerically in [20] that for any value of $\varepsilon$ there are intervals of $\mu$ values for which the symmetric orbit is elliptic. Here, we generalize this example to the $n$ dimensional case, for arbitrary large $n$, proving, that for certain classes of smooth repelling potentials (such as the power-law family) the smooth symmetric orbit which enters the vicinity of an $n$-corner has, for arbitrary small $\varepsilon$, intervals of $\mu$ values for which it is elliptic (all its $2 n$ multipliers belong to the unit circle). Furthermore, these intervals converge to positive $\mu$ values and their length, for sufficiently small $\varepsilon$ values scales as a function of $\varepsilon n$. In other words, we show that for arbitrarily large $n$, we can construct $n$-dimensional Sinai billiards and corresponding families of billiard-like smooth potentials, where, for arbitrary steepness the smooth flow possesses elliptic behavior. Our main result may be summarized by the following theorem:

Theorem 1. There exist families of analytic billiard potentials which limit (in the sense of [35]), as the steepness parameter $\varepsilon \rightarrow 0$, to Sinai billiards in $n$-dimensional compact domains $^{6}$, yet, for arbitrary small $\varepsilon$, the corresponding smooth Hamiltonian flows have stable (elliptic) periodic orbits.

Proof. We construct specific families of $n$-dimensional billiards depending on a parameter $\mu$, such that the billiards are Sinai billiards for any $\mu>0$ depending smoothly on $\mu$ for $\mu \in(0,1)$ (Section 2). We then consider families of potentials $W(x ; \mu, \varepsilon)$ which limit as $\varepsilon \rightarrow 0$, for any fixed $\mu$, to these billiards. We establish that for sufficiently small $\varepsilon$ these Hamiltonian flows have a periodic orbit $\gamma(t, \mu, \varepsilon)$ and we prove that the Floquet multipliers of this orbit may be found by solving a linear second order equation with a time-periodic coefficient (Section 3). This coefficient depends on $\mu, \varepsilon$ and $n$ as parameters, and it approaches a sum of delta-like functions as $\varepsilon \rightarrow 0$. For certain classes of $W(x ; \mu, \varepsilon)$ (e.g. when $W(x ; \mu, \varepsilon)$ decays as a power-law in the distance to the scatterers) we are able to analyze the asymptotic behavior of the emerging linear second order equation: we prove that for these potentials there are countable infinity values of $\mu$, one of them given by $\frac{1}{\sqrt{n}}$ (i.e. bounded away from $\mu=0,1$ ), from which a wedge of stability region in the $(\mu, \varepsilon)$ plane emerges. Namely, we prove that for any $n$, for arbitrary small $\varepsilon$, there exists an interval of $\mu$ values at which $\gamma(t, \mu, \varepsilon)$ is linearly stable (Lemma 1 in Section 3).

In particular, this theorem proves that such systems are not partial hyperbolic.

The paper is ordered as follows; we first construct the geometry of the limiting billiard domain. The construction of the billiards boundary, by intersecting several $(n-1)$ dimensional spheres in $R^{n}$, is valid for any finite dimension $n$. Then, we establish that in the smooth case, for sufficiently small $\varepsilon$, there exists a symmetric periodic orbit $\gamma(t)$ which corresponds to the one dimensional motion along the diagonal (in the $n$-dimensional space), and that this motion may be found by integrating a one-degree of freedom system which is independent of $n$. Next we show that the linear stability analysis about this motion is governed by a single second order linear differential equation with a time periodic coefficient in which $n$ appears as a parameter. In the third section we construct asymptotic solutions to this equation showing that for small $\varepsilon n$ it has intervals of parameter values at which $\gamma(t)$ is linearly stable, thus establishing the main theorem. Precise estimates of the length of these intervals are found for the power-law case. In the last section we integrate numerically these equations and compare the numerically found wedges of stability with the corresponding asymptotic estimates. Finally, we demonstrate the appearance of islands of

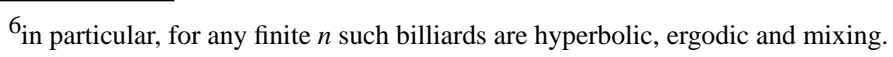



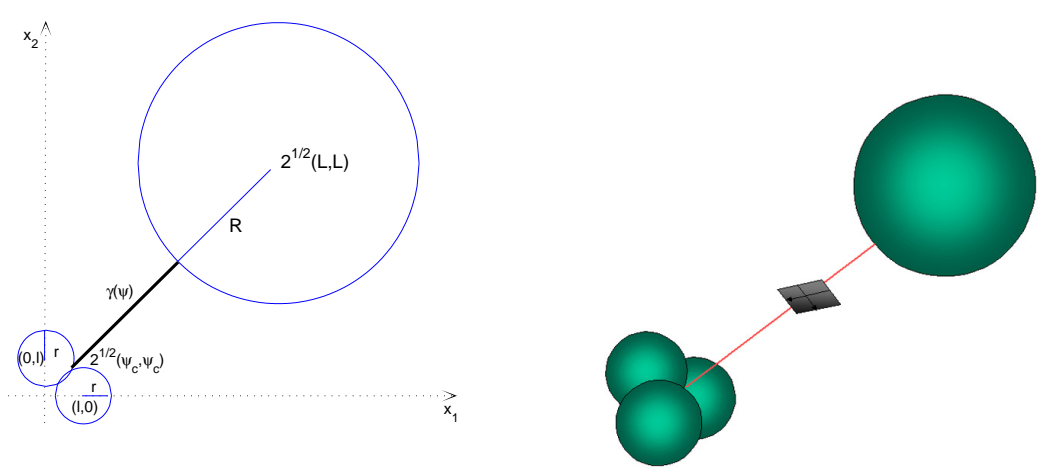

FIGURE 1. The billiard geometry in the two-dimensional and threedimensional cases.

effective stability by numerical integration of the symmetric $n$ d.o.f. system and of a slight asymmetric perturbation of it for a few $n$ values $(n=2,3,10)$ for two different types of potential families - the power-law family and the Gaussian family (e.g. we present islands of effective stability of dispersing, repelling, nonlinear 20 dimensional system).

\section{Construction OF THE BILliard AND THE LiMiting SMOOTH Flows}

2.1. The billiard geometry. Define the $n$-dimensional billiard's domain $D$ as the region exterior to $(n+1)$ spheres $S^{n-1}$ : one sphere $\Gamma_{n+1}$ of radius $R$ which is centered on the diagonal at a distance $L$ from the origin, i.e. at the point $\frac{1}{\sqrt{n}}(L, \ldots, L)$, and $n$ spheres $\Gamma_{1}, \ldots, \Gamma_{n}$ of radius $r$, each centered along a different principle axis at a distance $0 \leq l \leq r \sqrt{\frac{n}{n-1}}$ from the origin, i.e. the sphere $\Gamma_{k}$ is centered at $(\underbrace{0, \ldots, l}_{k}, \ldots, 0)$ (Figure 1). To obtain a bounded domain, we enclose this construction by a large $n$-dimensional hyper-cube centered at the origin (we will look only at the local behavior near the diagonal connecting the radius- $r$ spheres $\Gamma_{1}, \ldots, \Gamma_{n}$ to the radius- $R$ sphere $\Gamma_{n+1}$ and thus we will not be concerned with the form of the outer boundary). The diagonal line $(\xi, \ldots, \xi)$ intersects the radius$R$ sphere in the normal direction and the spheres $\Gamma_{1}, \ldots, \Gamma_{n}$ at their common intersection point $P_{c}=\left(\xi_{c}, \ldots, \xi_{c}\right)$, where (Figure 1):

$$
\xi_{c}=\frac{l}{n}+\frac{1}{\sqrt{n}} \sqrt{r^{2}-l^{2}\left(1-\frac{1}{n}\right)}
$$

Thus, for $L>R+\sqrt{n} \xi_{c}$, it defines a corner ray

$$
\gamma=\left\{(\xi, \ldots, \xi) \mid \xi \in\left(\xi_{c}, \frac{L-R}{\sqrt{n}}\right)\right\}
$$

that starts at the corner $P_{c}$, gets reflected from the radius- $R$ sphere and returns to $P_{c}$ (and then get stuck as there is no reflection rule at the corner).

Notice that the dynamics in the billiard is unchanged when all the geometrical parameters are proportionally increased, hence, with no loss of generality, we may set $r=1$ and regard all the other parameters as scaled by $r$. It is convenient for us to express the scaled 
$l$ and $L$ through

$$
\mu=\sqrt{1-\left(1-\frac{1}{n}\right) \frac{l^{2}}{r^{2}}} \text { and } d=\frac{L-R-\sqrt{n} \xi_{c}}{r} .
$$

Note that $\mu$ governs the angle created by the intersection of the $n$ spheres at the corner point; at $\mu=0$ the $n$ spheres are tangent to each other, namely the corner becomes a cusp. The case $\mu=1 / \sqrt{n}$ corresponds to $l=r$, hence the spheres intersect at a right angle. The case $\mu=1$ corresponds to $l=0$, namely the limit at which the $n$ spheres collapse to a single sphere of radius $r$ which is centered at the origin. In this case the diagonal becomes a hyperbolic periodic orbit of the billiard (note that the limit $\mu \rightarrow 1$ is singular: at $\mu=1$ the billiard's boundary is smooth, whereas for all $\mu \in(0,1)$ it has a corner).

2.2. Smooth motion - the diagonal periodic orbit. In this section we establish that for sufficiently small $\varepsilon$ the diagonal corner ray $\gamma$ of the billiard flow transforms into a periodic orbit of the smooth flow. Consider the smooth motion in the scaled billiard region, governed by the Hamiltonian (1.1), i.e.

$$
H=\sum_{i=1}^{n} \frac{p_{i}^{2}}{2}+W\left(x_{1}, \ldots, x_{n}\right)
$$

with

$$
W(x ; \varepsilon)=\frac{1}{n} \sum_{k=1}^{n} V\left(\frac{Q_{k}}{\varepsilon}\right)+V\left(\frac{Q_{n+1}}{\varepsilon}\right)
$$

where $Q_{k}(x)$ (the pattern function of $[23,21]$ ) is the distance from $x$ to $\Gamma_{k}$ :

$$
\begin{aligned}
& Q_{k}(x)=\sqrt{\sum_{i=1}^{n} x_{i}^{2}-2 l x_{k}+l^{2}}-1 \quad \text { for } k=1, \ldots, n \\
& Q_{n+1}(x)=\sqrt{\sum_{i=1}^{n}\left(x_{i}-\frac{L}{\sqrt{n}}\right)^{2}}-R
\end{aligned}
$$

(recall that we scale $r=1)$. The $C^{k+1}(k \geq 1)$ smooth function $V$ satisfies at $z>0$

$$
V(z)>0 \text { and } V^{\prime}(z)<0,
$$

so the potentials are repelling. We further assume that $V^{\prime \prime}(z)$ decays sufficiently rapidly for large $z$ (with accordance to the assumptions in $[23,21,35]$ ), so there exists some $\alpha>0$ such that

$$
V^{\prime \prime}(z)=O\left(\frac{1}{z^{2+\alpha}}\right) \text { as } z \rightarrow+\infty .
$$

As a typical $V$, one can take the power-law potentials:

$$
V(z)=\left(\frac{1}{z}\right)^{\alpha}, \alpha>0,
$$

the Gaussian potential

$$
V(z)=\exp \left(-z^{2}\right)
$$

or the exponential potential

$$
V(z)=\exp (-z)
$$

which naturally appear in applications (e.g. the Gaussian form arises in the problem of cold atomic motion in optical traps [10], whereas the power-law and exponential potentials are abundant in various classical models of atomic interactions). 
The potential $W(x ; \varepsilon)$ given by $(2.4),(2.5)$ is symmetric with respect to any permutation of the $x_{i}$ 's $(i=1, \ldots, n)$. This strong symmetry enables us to progress with the analysis for any $n$. Notice that it is easy to break this symmetry, by, for example, multiplying the terms $V\left(Q_{k}(x) / \varepsilon\right)$ in $(2.4)$ by slightly different coefficients. Such a modification is studied numerically in Section 4.2.

Now, consider the smooth motion along the diagonal $x_{1}=\cdots=x_{n}=\xi$. By the symmetry,

$$
\frac{\partial}{\partial x_{1}} W(\xi, \ldots, \xi)=\frac{\partial}{\partial x_{i}} W(\xi, \ldots, \xi) \text { for } i=1, \ldots, n \text {. }
$$

so the plane $\left\{x_{1}=\cdots=x_{n}=\xi, p_{1}=\cdots=p_{n}=\dot{\xi}\right\}$ is an invariant submanifold of the phase space. It follows from the conservation of energy that

$$
H=n \frac{\dot{\xi}^{2}}{2}+W(\xi, \ldots, \xi)
$$

for the orbits in this manifold; by differentiating this identity we obtain the following equation of motion on the invariant plane:

$$
\ddot{\xi}+\frac{\partial}{\partial x_{1}} W(\xi, \ldots, \xi)=0 .
$$

Let

$$
v=\sqrt{n}\left(\xi-\xi_{c}\right)
$$

where $\xi_{c}$ is defined by (2.1) with $r=1$. The energy conservation law (2.10) at the fixed energy level $h / 2$ reads as

$$
\frac{h}{2}=\frac{\dot{\mathrm{v}}^{2}}{2}+W_{e f f}(\mathrm{v} ; \varepsilon, \mu, d)
$$

where the effective potential is as follows (see (2.4),(2.5) and (2.12)):

$$
W_{e f f}=V\left(\frac{\sqrt{1+2 \mu \nu+v^{2}}-1}{\varepsilon}\right)+V\left(\frac{d-v}{\varepsilon}\right) .
$$

Equation (2.11) for the motion on the diagonal line transforms then into the equation (which is independent of $n$ ):

$$
\ddot{v}+\frac{\partial}{\partial \nu} W_{e f f}(\nu ; \varepsilon, \mu, d)=0 .
$$

This is a Hamiltonian equation with the Hamiltonian given by the right-hand side of (2.13). Since $V^{\prime}<0$, for any finite $\varepsilon$, the potential $W_{e f f}(v ; \varepsilon, \mu, d)$ has a minimal value for $v$ in the interval $(0, d)$ and the potential is monotonically increasing as the boundaries of this interval are approached. Thus, by (2.13), it has periodic solutions for the non-critical values of $h$ in the interval:

$$
h>h_{\min }(\varepsilon, \mu, d):=2 \min W_{e f f}(v)
$$

(at $h=h_{\min }$ the periodic orbit degenerates into an equilibrium point). The critical values of $h$ are those at which $W_{\text {eff }}$ has maxima, and then the periodic orbit is replaced by homoclinic or heteroclinic orbits.

Summarizing, we have established the following lemma: 
Lemma 1. For every non-critical value of $h>h_{\text {min }}(\varepsilon, \mu, d)$ the Hamiltonian flow (2.3) satisfying (2.4)-(2.6) possesses in the energy level $H=\frac{h}{2}$ a periodic solution of the diagonal form: $\gamma(t)=(\xi(t), \ldots, \xi(t))$ where $\xi(t)=\frac{v(t)}{\sqrt{n}}+\xi_{c}$ with $v(t) \in(0, d)$ being a periodic solution of (2.15) with energy $\frac{h}{2}$.

Let $T(\varepsilon, \mu, d, h)$ be the period of $\gamma(t)$. To fix the notation, let us parameterize time along $\gamma(t)$ so that $t=0$ will correspond to the turning point near the corner whereas $T / 2$ corresponds to the turning point near the large sphere, namely:

$$
W_{e f f}(v(0))=W_{e f f}(v(T / 2))=\frac{h}{2}
$$

with $v(0) \approx 0, v(T / 2) \approx d$.

\section{STABILITY OF THE PERIODIC ORBIT}

To study the stability of the periodic orbit $\gamma(t)$, one needs to linearize the Hamiltonian equations of motion corresponding to (1.1) about this solution, solve the corresponding $2 n$ dimensional linear system with the time-periodic coefficients for a set of $2 n$ orthonormal initial conditions and find the stability of the associated $(2 n \times 2 n)$-dimensional monodromy matrix, leading finally to a set of $2 n$ Floquet multipliers ( 2 of which are trivially one). The symmetric form of the potential allows to reduce this formidable task to a much simpler one - to solving a single second order homogeneous equation with a time periodic coefficient which depends on $n$ as a parameter in a very simple form:

Lemma 2. The Floquet multipliers of the T-periodic orbit $\gamma(t)$ are $\left(1,1, \lambda, \frac{1}{\lambda}, \ldots, \lambda, \frac{1}{\lambda}\right)$ where $\lambda$ is given by:

$$
\lambda=\frac{1}{2} \operatorname{Tr}(A)+\sqrt{\frac{\operatorname{Tr}(A)^{2}}{4}-1},
$$

and $A$ is the monodromy matrix of the second order linear equation:

$$
\ddot{y}+a(t) y=0
$$

with the T-periodic coefficient a $(t)$ given by (see also (2.15)):

$$
\begin{gathered}
a(t ; \varepsilon, \mu, d, R, n, h)=\left(\frac{V^{\prime}\left(\varepsilon^{-1}\left(\sqrt{1+2 \mu \nu+v^{2}}-1\right)\right)}{\varepsilon \sqrt{1+2 \mu v+v^{2}}}+\frac{V^{\prime}\left(\varepsilon^{-1}(d-v)\right)}{\varepsilon(R+d-v)}\right) \\
+\frac{1-\mu^{2}}{n-1}\left(\frac{V^{\prime \prime}\left(\varepsilon^{-1}\left(\sqrt{1+2 \mu \nu+v^{2}}-1\right)\right)}{\varepsilon^{2}\left(1+2 \mu v+v^{2}\right)}-\frac{V^{\prime}\left(\varepsilon^{-1}\left(\sqrt{1+2 \mu \nu+v^{2}}-1\right)\right)}{\varepsilon \sqrt{\left(1+2 \mu v+v^{2}\right)^{3}}}\right) \\
=a^{-}(v(t) ; \varepsilon, \mu, d, R, h)+\frac{1-\mu^{2}}{n-1} a^{+}(v(t) ; \varepsilon, \mu, d, h),
\end{gathered}
$$

Proof. Consider the linearization about $\gamma(t)$ of the system defined by (2.3). Let:

$$
\begin{aligned}
& b(t)=\frac{\partial^{2}}{\partial x_{1} \partial x_{2}} W(\xi(t), \ldots, \xi(t)), \\
& a(t)=\frac{\partial^{2}}{\partial x_{1}^{2}} W(\xi(t), \ldots, \xi(t))-b(t)
\end{aligned}
$$


By symmetry, $\frac{\partial^{2}}{\partial x_{i} \partial x_{j}} W(\xi(t), \ldots, \xi(t))=b(t)$ for all $i \neq j$ and $\frac{\partial^{2}}{\partial x_{i}^{2}} W(\xi(t), \ldots, \xi(t))=a(t)+$ $b(t)$ for all $i$. Hence,the linearization of (2.3) is given by

$$
\ddot{x}_{i}+a(t) x_{i}+b(t) \sum_{j=1}^{n} x_{j}=0, \quad i=1, \ldots, n .
$$

Let $s=\sum_{i=1}^{n} x_{i}$ and $y_{i}=x_{i}-\frac{s}{n}$ in (3.5). By summing the above equation on $i$ we obtain

$$
\begin{gathered}
\ddot{s}+(a(t)+n b(t)) s=0, \\
\ddot{y}_{i}+a(t) y_{i}=0 \quad i=2, \ldots, n
\end{gathered}
$$

Every equation in this system is decoupled from the others, therefore the spectrum of the Floquet multipliers of $\gamma(t)$ is the union of the spectra of the monodromy matrices (i.e. the spectra of the time- $T$ maps) corresponding to each of the equations. It is easy to check that the first equation is the linearization of (2.11) about $\xi(t)$. Hence, both the eigenvalues of its monodromy matrix are equal to 1 (as (2.11) is a Hamiltonian equation). These correspond to trivial Floquet multipliers of $\gamma(t)$. Since the rest of the equations in (3.6) are identical, the other Floquet multipliers of $\gamma(t)$ correspond to the $n-1$ identical pairs $\lambda$ and $\lambda^{-1}$, the eigenvalues of the monodromy matrix of the equation (3.2) with the $T$-periodic $a(t)$ given by (3.4). By applying the above formulas to the system (2.3),(2.4),(2.5), and using the coordinate $v$ instead of $\xi$ (see (2.12)), we obtain (3.3).

To establish the main theorem, the spectral properties of the $2 \times 2$ monodromy matrix $A$ of equation (3.2), that depend on $n$ and the geometric parameters via $a(t)$, need to be studied. The rest of this section is dedicated to estimating the eigenvalues of $A$ in various limits.

First, we show that in the limit of fixed $\varepsilon$ and large $n$ the periodic orbit $\gamma(t)$ is unstable. Likewise, we show that in the limit of low energies (near $h=h_{\min }(\varepsilon, \mu, d)$, see (2.16)), the periodic orbit which oscillates near the fixed point is unstable for all $n$ above some critical value. These observations show that the stable orbits we get do not correspond to a motion near the bottom of a potential well. Then, we prove the main result, that for any fixed $n$ there exists a sequence of $\mu$ values, $\mu_{k}$, such that the periodic orbit is stable in wedges in the $(\mu, \varepsilon)$ plane that are close to $\left(\mu_{k}, 0\right)$. The widths of these wedges is then found in two specific limits, with explicit formulae in the power-law potential case.

In the limit $n=+\infty$, equation (3.2) turns into

$$
\ddot{y}+a^{-}(t) y=0 .
$$

Since $a^{-}$is always negative by (2.6), this equation cannot have non-trivial bounded solutions and the monodromy matrix $A$ has multiplier $\lambda>1$. Thus, at every fixed $\varepsilon$ and $h>h_{\min }(\varepsilon, \mu, d)$, the diagonal solution $\gamma(t)$ is linearly unstable for sufficiently large $n$. Therefore, it is not surprising that the stability zones which we find later on correspond to bounded values of $\varepsilon n$, i.e. for higher dimension of the configuration space one should make the potential steeper in order to make the diagonal periodic orbit stable.

The stability of the equilibrium state on the diagonal, at $h=h_{\min }$, is determined by equation (3.2) of Lemma 2; the equilibrium is linearly stable if $a^{-}+\frac{1-\mu^{2}}{(n-1)} a^{+}>0$, and linearly unstable if $a^{-}+\frac{1-\mu^{2}}{(n-1)} a^{+}<0$, where instead of $v(t)$ in $a^{ \pm}$one should substitute 

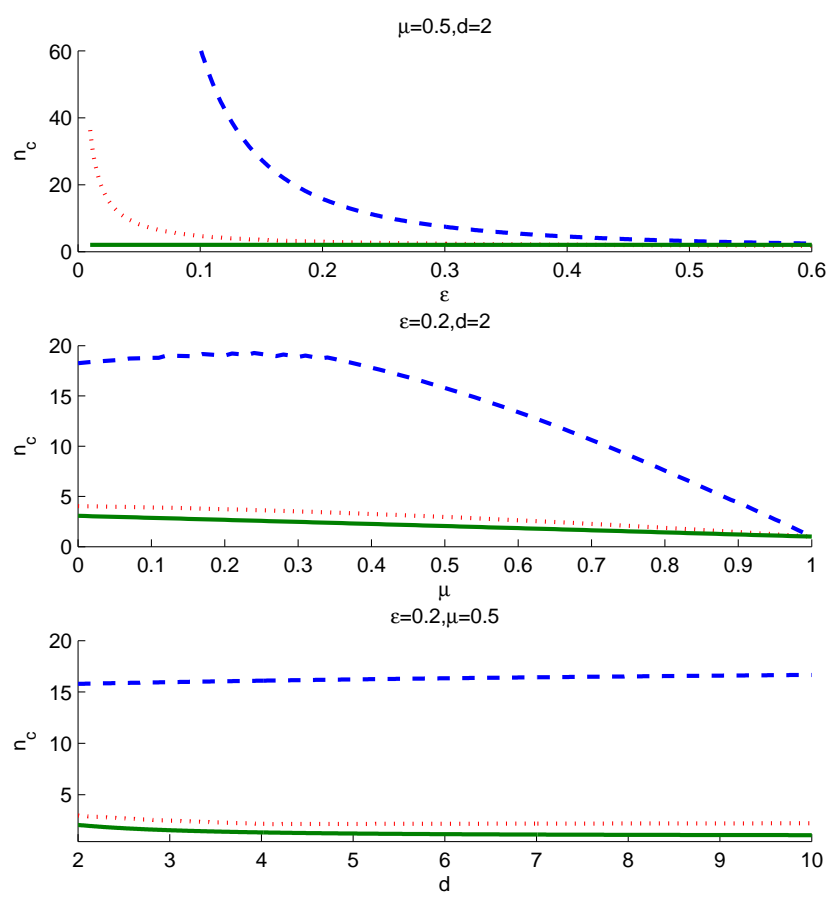

FIGURE 2. The critical dimension, $n_{c}$, beyond which the fixed point at the minimal energy level becomes unstable, for various $(\mu, d, \varepsilon)$ at $R=10$. Results for three potentials are presented: power-law (solid), exponential (dotted) and Gaussian (dashed).

the value of $v=v^{f}$ that corresponds to the minimum of $W_{\text {eff }}$ (see (2.14)). Defining

$$
n_{c}(\mu, d, R, \varepsilon)=1+\frac{a^{+}\left(v^{f}\right)}{-a^{-}\left(v^{f}\right)}\left(1-\mu^{2}\right),
$$

we see that the equilibrium (and small oscillations on the diagonal near it) are stable at $n<n_{c}$ and unstable at $n>n_{c}$. In Figure 2 we plot $n_{c}(\mu, d, R, \varepsilon)$ for the power-law, exponential and Gaussian potentials, showing the dependencies of $n_{c}$ on $\mu, d$ and $\varepsilon$. In the case of power-law potential, $n_{c}$ does not depend on $\varepsilon$ (see (2.8),(2.14) and (3.3)), thus, the stable periodic orbit that we find for small $\varepsilon$ clearly does not inherit its stability from the equilibrium state, i.e. the effect has truly billiard origin. For the exponential and Gaussian cases $n_{c}$ diverges as $\varepsilon \rightarrow 0$. In these cases the stable fixed point appears for exponentially small energies (see $((2.14))$. Since the effective potential is essentially flat away from the scatterers, for energies that are not exponentially small, the amplitude of the oscillations becomes large and the linearization near $v^{f}$ is not applicable. Indeed, it is proved below that for such energies the periodic orbit changes its stability several times as $\varepsilon \rightarrow 0$, so again, the stability regions we find do not correspond to small oscillations that inherit their stability from the equilibrium state. 

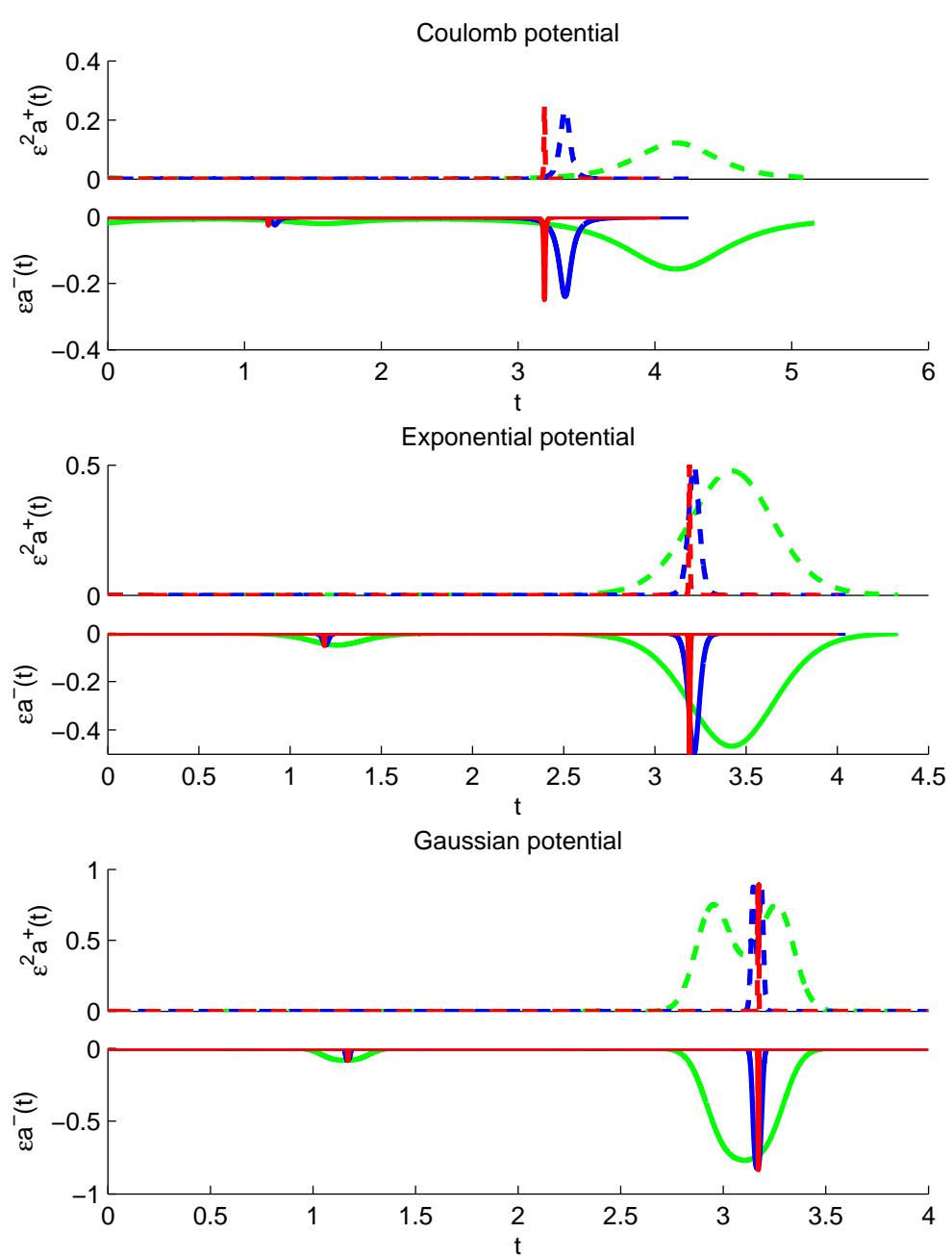

FIGURE 3. The rescaled ingredients of $a(t)$.The peaks of $a^{+}(\psi(t))$ and $a^{-}(\psi(t))$ are shown to scale as $1 / \varepsilon^{2}$ and $1 / \varepsilon$ respectively. Here $\mu=0.5$, and $\varepsilon=0.1,0.01,0.001$ from widest to narrowest respectively.

For any finite $n$, for sufficiently small $\varepsilon, \gamma(t)$ has a finite positive period and $a(t)$ changes $\operatorname{sign}^{7}$ as shown in Figure 3, so the behavior of the monodromy matrix $A$ in the limit $\varepsilon \rightarrow 0$ becomes non-trivial. Our main result is that there are wedges in the $(\mu, \varepsilon)$ space at which the eigenvalues of $A$ are on the unit circle:

\footnotetext{
${ }^{7}$ While $a^{-}$is always negative, for sufficiently small $\varepsilon$, there exists an interval of $t$ values at which $a^{+}$is positive (as $V^{\prime}$ is negative, and $V$ is bounded from below, it follows that $V^{\prime \prime}$ has to be positive somewhere). In fact, $a^{+}>0$ everywhere in the power-law potential case.
} 
Theorem 2. Suppose the potential function $V$ satisfies (2.6),(2.7). Then, given any $h \in$ $(0,2 V(0))$, any natural $n \geq 2$, and any positive $d$ and $R$, there exists a tending to zero countable infinite sequence $1 \geq \mu_{0}>\mu_{1}=1 / \sqrt{n}>\ldots>\mu_{k}>\ldots>0$ such that arbitrarily close to every point $\left(\mu=\mu_{k}, \varepsilon=0\right)$ there are wedges of $(\mu, \varepsilon)$ at which the orbit $\gamma$ is linearly stable.

Proof. Recall the definition of the monodromy matrix $A$ : the linear second order differential equation (3.2) with the periodic coefficient $a(t)$ defines the linear map: $\left(y\left(t_{0}\right), y^{\prime}\left(t_{0}\right)\right) \mapsto$ $\left(y\left(t_{0}+T\right), y^{\prime}\left(t_{0}+T\right)\right)=A\left(y\left(t_{0}\right), y^{\prime}\left(t_{0}\right)\right)$. While $A$ may depend on the choice of $t_{0}$, its eigenvalues, the Floquet multipliers of $\gamma(t)$, do not. We choose $t_{0}=-\Delta t$ where $\Delta t>0$ is slowly tending to zero as $\varepsilon \rightarrow 0$, and express $A$ as the product of two matrices: $A=B C$, where $C$ corresponds to the map from $t=-\Delta t$ to $t=\Delta t$ (i.e. to the linearized smooth motion in the neighborhood of the billiard corner), and $B$ corresponds to the time interval $[\Delta t, T-\Delta t]$ (i.e. to the linearization about the smooth regular motion along the diagonal and the regular reflection from the radius $R$ sphere $\Gamma_{n+1}$ in the normal direction at $t=T / 2$ ).

Below, we find the form of $B$ and $C$ in the limit of small $\varepsilon$ and fixed $\mu$. We then compute the trace of $A$ and find the wedges in the $(\mu, \varepsilon)$ plane, where the trace of $A$ varies between -2 to 2 .

The form of $B$ is easily found by utilizing the billiard limit (using [21]) :

Lemma 3. For small $\Delta t$ and sufficiently small $\varepsilon$, the linearized map about the diagonal orbit: $\left(y(\Delta t), y^{\prime}(\Delta t)\right) \mapsto\left(y(T-\Delta t), y^{\prime}(T-\Delta t)\right)=B\left(y(\Delta t), y^{\prime}(\Delta t)\right)$ satisfies

$$
B=\left(\begin{array}{cc}
1+\frac{2 d}{R} & \frac{2 d}{\sqrt{h}}\left(1+\frac{d}{R}\right) \\
\frac{2}{R} \sqrt{h} & 1+\frac{2 d}{R}
\end{array}\right)+o(1)
$$

Proof. Fixing $\Delta t$ and letting $\varepsilon \rightarrow 0$, the diagonal periodic orbit $\gamma(t)$ on the interval $[\Delta t, T-$ $\Delta t$ approaches the boundary of the billiard domain only once, at $t=T / 2$, hitting the radius$R$ sphere $\Gamma_{n+1}$ in the normal direction. This is a regular reflection, therefore, according to $[21]^{8}$, the flow map from any time moment before the reflection to any moment after the reflection is close to the corresponding map for the billiard flow. The closeness is along with $k$ derivatives of the map (recall that $V$ is $C^{k+1}, k \geq 1$ ), i.e. the derivative of the flow map from $t=\Delta t$ to $t=T-\Delta t$ tends to the derivative of the billiard flow map as $\varepsilon \rightarrow 0$. It is true for every fixed $\Delta t$, hence it remains true for a sufficiently slowly tending to zero $\Delta t$.

Because of the symmetry of the diagonal orbit $\gamma$, the matrix of the derivative of the smooth flow has a block-diagonal structure with one idempotent block that corresponds to the variable $s$ in (3.6) and the other blocks equal to $B$. The derivative matrix of the billiard flow has the same structure; to find this matrix, consider the billiard flow of a particle with a velocity $\sqrt{h}$ which starts at a distance $d$ from the sphere of radius $R$ and reflects in the normal direction back to its original position at $T=2 d / \sqrt{h}$. Then, by direct computation, it can be shown that the $i$ th block of the linearization of the billiard flow map is of the form:

$$
\frac{\partial\left(y_{i}(T-0), \dot{y}_{i}(T-0)\right)}{\partial\left(y_{i}(+0), \dot{y}_{i}(+0)\right)}=\left(\begin{array}{cc}
1+\frac{2 d}{R} & \frac{2 d}{\sqrt{h}}\left(1+\frac{d}{R}\right) \\
\frac{2}{R} \sqrt{h} & 1+\frac{2 d}{R}
\end{array}\right)
$$

and (3.7) follows from [21] as explained above (the same results can be achieved by asymptotic integration of equation (3.2), namely following a simplified version of the below construction of $C$ ).

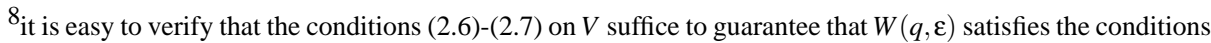
in [21].
} 
Finding the form of $C$ is more complicated, and requires the integration of (3.2) in some asymptotic limits. In Appendix A, we prove the following:

Lemma 4. For any fixed $\mu \in(0,1)$, small $\Delta t$ and sufficiently small $\varepsilon$, the linearized map about the diagonal orbit near the corner: $\left(y(-\Delta t), y^{\prime}(-\Delta t)\right) \mapsto\left(y(\Delta t), y^{\prime}(\Delta t)\right)=C\left(y(\Delta t), y^{\prime}(\Delta t)\right)$ satisfies

$$
C=\left(\begin{array}{cc}
s_{11}+\sigma s_{21}(1+o(1))+o(1) & s_{21} O\left(\delta \sigma^{2}\right)+O(\delta \sigma) \\
\frac{1}{\delta}\left(s_{21}(1+o(1))+O\left(\sigma^{-1-\alpha}\right)\right) & s_{22}+\sigma s_{21}(1+o(1))+o(1)
\end{array}\right),
$$

where $\delta, \sigma^{-1}$ are small scaling parameters (tending to 0 as $\varepsilon \rightarrow 0$ ) such that $\delta \sigma=\Delta t$, and $S$ is a matrix which tends ${ }^{9}$, as $\varepsilon \rightarrow 0$, to a smooth limit $S_{0}(\mu)$.

Let us explain the meaning of the matrix $S$ which appears in (3.8). It is shown that in the appropriate scaling limit equation (3.2) near the corner reduces to

$$
\frac{d^{2}}{d \tau^{2}} y+\beta V^{\prime \prime}(z(\tau)) y=0,
$$

where

$$
\beta=\frac{1-\mu^{2}}{(n-1) \mu^{2}}
$$

and $z(\tau)$ solves

$$
\frac{h}{2}=\frac{\left(z^{\prime}\right)^{2}}{2}+V(z), z^{\prime}(0)=0
$$

Notice that by (2.7) equations (3.9)-(3.11) define a scattering matrix: it is shown in the appendix that the solutions $z(\tau)$ to (3.11) run from $+\infty$ through some minimal positive value back to $+\infty$ sufficiently rapidly and thus that (3.9) reduces, in the limit of $\tau \rightarrow \pm \infty$ to $\frac{d^{2}}{d \tau^{2}} y=0$. Then, as is usual in scattering theory, one may define two bases of solutions at the two asymptotic limits. Let $y_{ \pm}(\tau)$ denote the uniquely defined solutions having the following asymptotic form as $\tau \rightarrow \pm \infty$ (respectively):

$$
y_{ \pm}(\tau)=1+O\left(|\tau|^{-\alpha}\right), \quad y_{ \pm}^{\prime}(\tau)=O\left(|\tau|^{-1-\alpha}\right) .
$$

Let $\left.\widehat{y}_{ \pm}(\tau)\right)$ denote solutions ${ }^{10}$ with asymptotic:

$$
\hat{y}_{ \pm}=\tau+O\left(|\tau|^{1-\alpha}\right), \quad y_{ \pm}^{\prime}(\tau)=1+O\left(|\tau|^{-\alpha}\right)
$$

so the Wronskians of $\left(y_{-}(\tau), \widehat{y}_{-}(\tau)\right)$ and of $\left(y_{+}(\tau), \widehat{y}_{+}(\tau)\right)$ are 1. Let $S_{0}$ denote the scattering matrix which sends the coefficients of the solution in the basis $\left(y_{-}(\tau), \widehat{y}_{-}(\tau)\right)$ into the coefficients of the same solution in the basis $\left(y_{+}(\tau), \widehat{y}_{+}(\tau)\right)$. This matrix depends only on $\beta$ and $h$ - the only two parameters which appear in the above limit equations. In Appendix A, we derive the finite $\varepsilon$ version of (3.9)-(3.11), the corresponding asymptotic bases and the scattering matrix $S(\mu, \varepsilon)$ which limits to $S_{0}(\mu)$ as $\varepsilon \rightarrow 0$ for any fixed $\mu>0$.

Using the formulae for $B$ and $C((3.7)$ and (3.8)) with $\sigma \delta=\Delta t$ tending to zero sufficiently slowly and $\delta \rightarrow 0$, one obtains that the trace of the monodromy matrix $A=B C$ equals to

$$
\operatorname{Tr}(A)=\frac{2 d}{\delta \sqrt{h}}\left(1+\frac{d}{R}\right) s_{21}(1+o(1))+\left(s_{11}+s_{22}\right)\left(1+\frac{2 d}{R}\right)+o(1) .
$$

The periodic orbit is stable when $|\operatorname{Tr}(A)|<2$. Note that the main contribution to (3.14) is given by the term that includes $s_{21}$ : since $\delta \rightarrow 0$ as $\varepsilon \rightarrow 0$, if $s_{21}(\mu, \varepsilon)$ stays bounded away

\footnotetext{
${ }^{9}$ uniformly on any compact subset of $\mu>0$.

10 the functions $\left.\widehat{y}_{ \pm}(\tau)\right)$ are defined in a unique way in Appendix A.
} 
from zero, then for sufficiently small $\varepsilon$ the trace of $A$ is very large and positive for positive $s_{21}$ and very large negative for negative $s_{21}$. This means that if we fix $h, n, d, R$, choose $\varepsilon$ sufficiently small and change $\mu$, then $\operatorname{Tr}(A)$, as a function of $\mu$, will change sign near the values of $\mu$ where $s_{21}(\mu, 0)$ changes sign. $\operatorname{Then} \operatorname{Tr}(A)$ is necessarily small near these values of $\mu$. Therefore, from these values of $\mu$ a wedge of parameter values for which the periodic orbit $\gamma$ is linearly stable emerges. We need to establish that there is an infinite number of such values of $\mu$.

By definition, $s_{21}$ is determined as follows (see appendix): take the solution $y_{-}(\tau)$ of (3.9) that tends to 1 as $\tau=-\infty$, then

$$
s_{21}=\frac{d y_{-}}{d \tau}(+\infty)
$$

namely, the asymptotic properties of $y_{-}(\tau)$ determine $s_{21}$. Next we establish:

Lemma 5. The limit system (3.9) has a non trivial bounded solution $y_{-}\left(\tau ; \mu^{*}\right)$ for all $\tau \in(-\infty,+\infty)$ if and only if $s_{21}\left(\mu^{*}, 0\right)=0$. Furthermore,

$$
\left.\frac{d s_{21}}{d \beta}\right|_{s_{21}=0}= \begin{cases}-\sqrt{h} I, & \beta\left(\mu^{*}\right)=0, \\ -\frac{1}{\beta y_{-}(+\infty)} \int_{-\infty}^{+\infty} y_{-}^{\prime}(s)^{2} d s, & \beta\left(\mu^{*}\right) \neq 0,\end{cases}
$$

where

$$
I=\frac{1}{\sqrt{h}} \int_{-\infty}^{+\infty} V^{\prime \prime}(z(\tau)) d \tau=\frac{2}{\sqrt{h}} \int_{V^{-1}(h / 2)}^{+\infty} V^{\prime \prime}(z) \frac{d z}{\sqrt{h-2 V(z)}} .
$$

If $s_{21}(\mu, 0) \neq 0$, then sign $s_{21}=(-1)^{\mathcal{N}\left(y_{-}\right)}$where $\mathcal{N}\left(y_{-}\right)$denotes the number of zeroes of $y_{-}(\tau)$. Finally, if $\mu^{*}<1$, or $\mu^{*}=1$ and $I>0$, then $\mathcal{N}\left(y_{-}\right)$is decreased by one when $\mu$ changes from $\mu^{*}-0$ to $\mu^{*}+0$.

Proof. Using the definition of the scattering matrix (see (3.12),(3.13)), $y_{-}$has the following asymptotic as $\tau \rightarrow+\infty$ (uniformly on any compact subset of positive values of $\mu$ ):

$$
\begin{aligned}
& y_{-}=s_{21}\left(\tau+O\left(\tau^{1-\alpha}\right)\right)+s_{11}\left(1+O\left(\tau^{-\alpha}\right)\right), \\
& y_{-}^{\prime}=s_{21}\left(1+O\left(\tau^{-\alpha}\right)\right)+O\left(\tau^{-1-\alpha}\right),
\end{aligned}
$$

It thus follows immediately that if $s_{21}$ vanishes, then $y_{-}(\tau)$ is bounded. To prove the converse, notice that non-trivial bounded solutions must be proportional to $y_{-}(\tau)$ as $\tau \rightarrow-\infty$, and therefore, if $s_{21} \neq 0$, these cannot remain bounded as $\tau \rightarrow+\infty$.

Next, we establish (3.16). Define $u=d y_{-} / d \beta$. By definition (see (3.15))

$$
\frac{d s_{21}}{d \beta}=u^{\prime}(+\infty)
$$

By differentiating (3.9) with respect to $\beta$ we find that $u$ is the solution of

$$
u^{\prime \prime}+\beta V^{\prime \prime}(z(\tau)) u=-V^{\prime \prime}(z(\tau)) y_{-}(\tau)
$$

which satisfies $u(-\infty)=u^{\prime}(-\infty)=0$. By the variation of constants formula (recall that the Wronskian of $y_{-}(\tau)$ and $\hat{y}_{-}(\tau)$ is 1$)$ :

$$
u(\tau)=y_{-}(\tau) \int_{-\infty}^{\tau} V^{\prime \prime}(z(s)) y_{-}(s) \hat{y}_{-}(s) d s-\hat{y}_{-}(\tau) \int_{-\infty}^{\tau} V^{\prime \prime}(z(s)) y_{-}(s)^{2} d s .
$$

It follows that

$$
u^{\prime}(+\infty)=y_{-}^{\prime}(+\infty) \int_{-\infty}^{+\infty} V^{\prime \prime}(z(s)) y_{-}(s) \hat{y}_{-}(s) d s-\hat{y}_{-}^{\prime}(+\infty) \int_{-\infty}^{+\infty} V^{\prime \prime}(z(s)) y_{-}(s)^{2} d s
$$


If $s_{21}=0$, then we have $y_{-}^{\prime}(+\infty)=0$ and $\hat{y}_{-}^{\prime}(+\infty)=y_{-}^{-1}(+\infty)$ (since the Wronskian is 1). Thus,

$$
\frac{d s_{21}}{d \beta}=-\frac{1}{y_{-}(+\infty)} \int_{-\infty}^{+\infty} V^{\prime \prime}(z(s)) y_{-}(s)^{2} d s \text { at } s_{21}=0 .
$$

At $\beta \neq 0$ we have $V^{\prime \prime}(z) y^{-}=-\beta^{-1} y_{-}^{\prime \prime}$, hence, integrating by parts, we find

$$
\int_{-\infty}^{\tau} V^{\prime \prime}(z(s)) y_{-}(s)^{2} d s=\frac{1}{\beta} \int_{-\infty}^{+\infty} y_{-}^{\prime}(s)^{2} d s
$$

which gives the second line of (3.16).

At $\beta=0$ the scattering matrix of system (3.9) is the identity so $s_{21}(1,0)=0$. In this case (3.9) has the bounded solution $y(\tau)=1$ and by (3.21) the first line of (3.16) is obtained, or equivalently

$$
\left.\frac{d s_{21}}{d \mu}\right|_{\mu=1}=\frac{2}{n-1} \sqrt{h} I
$$

Finally, let us relate the number of zeroes of the fundamental solution $y_{-}, \mathcal{N}\left(y_{-}\right)$, and the sign of $s_{21}$. By (3.15), if $y_{-} \rightarrow+\infty$ as $\tau \rightarrow+\infty$, then $s_{21}>0$, and if $y_{-} \rightarrow-\infty$ as $\tau \rightarrow+\infty$, then $s_{21}<0$. Recall that $y_{-}(-\infty)=1$ is always positive. Clearly, if $s_{21}>0$, then $y_{-}$has an even number of zeros, and if $s_{21}<0$, then the number of zeros of $y_{-}$is odd so $\operatorname{sign} s_{21}=(-1)^{\mathcal{N}\left(y_{-}\right)}$as claimed.

Note that $y_{-}$cannot have multiple zeros, as it is a non-trivial solution of a second order linear homogeneous equation. It follows that as $\mu$ varies, the number of zeros of $y_{-}$can increase only when some zeros come out of $+\infty$.

It follows from (3.18), and the fact that $s_{21}^{2}+s_{12}^{2}$ is bounded away from zero by preservation of the Wronskian, that $y_{-}$may have only one zero at large $\tau$. Therefore, if $\mathcal{N}\left(y_{-}\right)$ changes at some $\mu>0$, the increase/decrease in the value of $\mathcal{N}$ equals exactly to 1 .

It follows from (3.20) and (3.18) that at $s_{21}=0$ (i.e. when $y_{-}(\tau)$ is bounded)

$$
\frac{d}{d \beta} y_{-}(\tau)=u(\tau)=\tau u^{\prime}(+\infty)+o(\tau)_{\tau \rightarrow+\infty}
$$

Hence, it follows from (3.19), and from (3.16) with $\mu^{*}<1$, or $\mu^{*}=1$ and $I>0$, that for all $\tau$ sufficiently large

$$
\operatorname{sign} \frac{d}{d \mu} y_{-}(\tau)=\operatorname{sign} \frac{d s_{21}}{d \mu}=\operatorname{sign} y_{-}(+\infty)=(-1)^{\mathcal{N}\left(y_{-}\right)} .
$$

so it follows that $\mathcal{N}\left(y_{-}\right)$decreases when $\mu$ increases through $\mu^{*}$.

It follows from the trace formula of $A$ (3.14), that to complete the proof of Theorem 2 , we need to show that the coefficient $s_{21}$ of the scattering matrix for the limit equation (3.9),(3.11) changes its sign infinitely many times. By the above lemma, we need to examine the bounded solutions of (3.9) and their number of zeroes.

Now, notice that independently of the choice of $V$ and of the value of $h$ there are two values of $\mu$ at which the bounded solutions are easily identified. At $\mu=1$ we have the bounded solution $y_{-}=1$ which has no zeroes. At $\mu=\mu_{1}=n^{-1 / 2}$ there is a bounded solution with one zero: $y(\tau)=z^{\prime}(\tau)$, where $z$ is the solution of (3.11). It follows from (3.16) that when ${ }^{11} I>0, s_{21}$ changes sign from negative to positive when $\mu$ increases

\footnotetext{
${ }^{11}$ This is always the case if $V^{\prime \prime}>0$ for all $z$, e.g. for the power-law potentials (2.8), where the following explicit formula for $I$ may be established:

$$
I=2(\alpha+1)(h / 2)^{1 / \alpha} \int_{0}^{\pi / 2}(\cos \theta)^{\frac{2}{\alpha}+1} d \theta>0 .
$$
}


through $\mu=1$ (recall that $\beta^{\prime}(\mu)<0$ for $\mu<1$ ), and when $I<0$ (this is the case e.g. of Gaussian potential (2.9) at $h$ close to 2) $s_{21}$ changes sign from positive to negative. It follows from (3.24) that at $\mu=\mu_{1}+0$ we have $s_{21}<0$. Hence, using (3.23) we see that if $I<0$, there exists $\mu=\mu_{0}<1$ for which $s_{21}=0$ (so there is a non-constant positive bounded solution at $\left.\mu_{0}\right)$. This is the tip of the 0 -th stability zone. Furthermore, since for $\mu_{k}<1$ the number of zeros of $y_{-}$always decreases by one when $\mu$ changes from $\mu_{k}-0$ to $\mu_{k}+0$, it follows that for $I<0$ there is only one such $\mu_{0}$ value in the interval $\left(n^{-1 / 2}, 1\right)$, whereas for $I>0$ we set $\mu_{0}=1$.

We conclude that for $k \geq 1$, the tip $\mu=\mu_{k}$ of the $k$-th stability zone corresponds to the existence of a bounded solution of (3.9), which has exactly $k$ zeros. To establish that there is a countable infinity of values of such $\mu_{k}$, recall that there is a non-empty interval of values of $\tau$ for which $V^{\prime \prime}(z(\tau))$ is strictly positive (by (2.6) and (2.7)). Since the coefficient $\beta$ of $V^{\prime \prime}(z) y$ grows to $+\infty$ as $\mu \rightarrow+0$, it follows that the number of zeros of every solution of (3.9) on this interval grows to infinity as $\mu \rightarrow+0$. In particular, the number of zeros of $y_{-}-$hence the number of sign changes in $s_{21}$ - grows to infinity as $\mu \rightarrow+0$, as required.

This completes the proof of Theorem 2 .

Notice that the points $\mu_{k}$ where the stability zones touch the axis $\varepsilon=0$ are determined by the behavior of the limit system (3.9)-(3.11) only. In particular, depending on the form of $V$ and $h$ there are the corresponding $\beta_{k}$ values at which the stability zones appear, and these are independent of $n, d$ and $R$. Thus, we conclude from (3.10) that

$$
\mu_{k}=\left(1+\beta_{k}(n-1)\right)^{-1 / 2},
$$

where the numbers $\beta_{k} \rightarrow+\infty$ depend only on $h$ and on the potential function $V$. If $I>0$ then $\beta_{0}=0$. For all $V$ and $h$ we have $\beta_{1}=1$.

Note that in the proof of Theorem 2 the limit of fixed $\mu>0$ and $\varepsilon \rightarrow 0$ was considered. It follows that for any finite $k$ value a stability zone will appear near $\mu_{k}$ for sufficiently small $\varepsilon$ (non-uniformly in $k$ ). In the appendix we prove that an infinite number of these stability zones extend towards the $\varepsilon$ axis:

Lemma 6. Let $\mathcal{L}$ be a continuous curve in the region $(\mu \geq 0, \varepsilon>0)$ of the $(\mu, \varepsilon)$-plane, which starts at $(\mu=0, \varepsilon=0)$. Then $\mathcal{L}$ intersects the region of stability of the diagonal periodic orbit $\gamma$ in an infinite sequence of intervals converging to $(\mu=0, \varepsilon=0)$.

Proof. See Appendix A. After calculating the form of the matrix $C$ in this limit of small $(\mu, \varepsilon)$, which involves deriving a rescaled system similar to (3.9), it is shown that the trace of $A$ changes between \pm 2 whenever the number of zeroes of the bounded solutions of this rescaled system are changed. Then, we again argue that the number of zeros of this system tends to infinity as $(\mu, \varepsilon) \rightarrow 0$.

3.1. Estimates of the stability wedges width. We have thus established that for any finite dimension $n$ there is a infinite number of wedges of linear stability zones emanating from $\mu$ values at $(0,1)$. Next we estimate their width in the $(\mu, \varepsilon)$-plane at $\beta$ values that are near $\beta_{0}=0$ (corresponding to either $\mu$ close to 1 or to large $n$ ):

Proposition 1. If I $>0$ (see (3.17)), then the diagonal periodic orbit $\gamma$ is stable for $(\mu, \varepsilon)$ values in the wedge enclosed by the two curves

$$
\varepsilon_{0}^{+}=I \frac{1-\mu^{2}}{(n-1) \mu^{2}}\left(1+\frac{1}{d+R}\right)^{-1}+o\left(\frac{1-\mu^{2}}{(n-1) \mu^{2}}\right)
$$


and

$$
\varepsilon_{0}^{-}=I \frac{1-\mu^{2}}{(n-1) \mu^{2}}\left(1+\frac{1}{d}\right)^{-1}+o\left(\frac{1-\mu^{2}}{(n-1) \mu^{2}}\right) .
$$

Proof. See Appendix A, where formula (3.14) is expanded in $\beta, \varepsilon$ near $(0,0)$ at which $S$ limits to the identity matrix.

The other limit in which we are able to obtain analytical results regarding the stability wedges width corresponds to $\mu=0$, i.e. it is the limit of the zero angle between the spheres $\Gamma_{1}, \ldots, \Gamma_{n}$ at the corner point. We prove that for sufficiently large $k$, the stability zone emanating from $\left(\mu_{k}, \varepsilon=0\right)$ extends towards the $\varepsilon$-axis as shown in Figure 4:

Proposition 2. Consider the power-law potential $V(Q, \varepsilon)=\left(\frac{\varepsilon}{Q}\right)^{\alpha}$. Then, for sufficiently small $\varepsilon$ and $\mu$, there exists an infinite number of disjoint stability tongues in the $(\mu, \varepsilon)$ plane at which $\gamma(t ; \mu, \varepsilon, n)$ is linearly stable. For sufficiently large $k$ the kth stability zone emanates from the $\mu$ axis near the bifurcation value:

$$
\mu_{k} \approx \frac{1}{k} \sqrt{\frac{2(\alpha+1)}{\alpha(n-1)}},
$$

and extends up to the $\varepsilon$-axis, intersecting it near

$$
\varepsilon_{k} \approx(h / 2)^{1 / \alpha} \frac{(\alpha+1)}{\alpha(n-1)} \frac{4}{\pi^{2} k^{2}}\left(\int_{0}^{\pi / 2}(\sin \theta)^{1 / \alpha} d \theta\right)^{2},
$$

at a stability interval of length

$$
(\Delta \varepsilon)_{k} \approx \frac{4 \varepsilon_{k}}{\pi k G(0, \alpha) d\left(1+\frac{d}{R}\right)}\left(\frac{4 \alpha(\alpha+1)}{n-1} \frac{\left(2 \varepsilon_{k}\right)^{\alpha}}{h}\right)^{1 / 2(\alpha+1)}
$$

where $G(0, \alpha)>0$ depends only on $\alpha$ and is defined by (B.12).

Proof. See Appendix B for details. It is proved that any curve of the form

$$
\mathcal{L}_{M}=\left\{(\mu, \varepsilon): 2 \varepsilon M=\mu^{2}(1-M)\right\},
$$

with $M \in[0,1]$ considered as a fixed parameter, intersects the stability wedges infinite number of times. Moreover, the location and width of these intersections is evaluated along a parametrization of $\mathcal{L}_{M}$ by an auxiliary parameter $\rho$ :

$$
\rho=\sqrt{2 \varepsilon+\mu^{2}} .
$$

Thus, formula (3.28) is established by applying formula (B.14) along $\mathcal{L}_{M=1}$ whereas formulae (3.29),(3.30) are established by applying (B.14) and (B.15) along $\mathcal{L}_{M=0}$ (using (B.3) and (B.5)).

As described next, the asymptotic formulae are in excellent agreement with our numerics. 


\section{NUMERICAL COMPUTATIONS}

4.1. Stability of the periodic orbit. In general, the numerical computation of a periodic trajectory and its stability in a steep $n$-dimensional potential is, for large $n$, a difficult problem; a high-dimensional scheme for locating the periodic trajectory is needed, and the search involves the integration of nonlinear, stiff, high-dimensional system. Once the periodic orbit is found, the numerical computation of the linearized system and its Floquet multipliers for large $n$ may be a formidable task.

Here we use Lemmas 1 and 2 and some proper rescaling to reduce this problem to a simpler computational task. The search for the periodic orbit is unnecessary by Lemma 1 (using symmetry and proper parameters) and the need to compute eigenvalues of large matrices is demolished by Lemma 2: for all $n$ we find the solutions of one second-order non-linear equation (2.15) and the monodromy matrix of one second-order linear equation (3.2),(3.3) which depends on $n$ as a parameter. The steep limit is handled as in [20]: we fix $\varepsilon$ and increase the size of the billiard domain ( $r$ in (2.2)) to get an effectively small $\bar{\varepsilon}=\varepsilon / r$ without running into stiffness problems (in the bulk of the domain the motion is essentially inertial and non-stiff).

To find the stability regions, as shown in Figure 4, we use the continuation scheme which was developed in [20]; first we compute the stability of $\gamma(t)$ at $\mu=0$ (the case of a cusp created by $n$ tangent spheres) along the $\varepsilon$-axis (see Figure 4 left). By symmetry (see Lemma 2), $\left.\operatorname{Re}\left(\mid \lambda_{n}(\mu=0, \varepsilon)\right) \mid\right)>1$ always corresponds to real eigenvalue (i.e. saddlefoci do not appear) and thus the values of $\varepsilon=\varepsilon_{k}^{ \pm}(n)$ at which $\operatorname{Re}\left(\lambda_{n}(\mu=0, \varepsilon)\right)= \pm 1$ correspond to degenerate saddle-center and degenerate period-doubling bifurcations respectively. Then, we use the values of $\varepsilon=\varepsilon_{k}^{ \pm}(n)$ as the starting point for a continuation scheme in $\mu$ to locate the $k$ th wedge of stability in the $(\mu, \varepsilon)$ plane (see Figure 4 right).

With accordance to Theorem 2 and Propositions 1,2, these calculations (performed for the Gaussian, exponential and power-law potentials, and shown here only for the powerlaw case) demonstrate that for any given $n$, at $\mu=0$, the stability of $\gamma_{\varepsilon, n, \mu=0}(t)$ rapidly changes as $\varepsilon \rightarrow 0^{+}$, whereas for any $\mu \in(0,1)$, there are finite number of intervals of $\varepsilon$ in which $\gamma_{\varepsilon, n, \mu}(t)$ is stable.

Next, we demonstrate that the asymptotic formulae provided in these propositions are in good agreement with the numerics; in all the numerical simulations shown below we fix $h=1, R=10$ and $d=2$, consider the power-law case (2.8) with $\alpha=1$, and study, for each $n$, how the stability of $\gamma(t)$ depends on $\mu$ and $\varepsilon$.

Figure 4 shows that the estimates (3.26),(3.27) of Proposition 1 for the stability boundary of the first wedge and their numerical calculation agree when either $1-\mu$ is small or $n$ is large (recall the $o\left(\frac{1-\mu^{2}}{(n-1) \mu^{2}}\right)$ correction term in (3.26),(3.27)).

The origin of the second stability zone at $\varepsilon=0$ is found, by Theorem 2 , to be given by $\mu_{1}=1 / \sqrt{n}$, so $\mu_{1}^{n=3} \approx 0.577$ and $\mu_{1}^{n=10} \approx 0.33$, which agrees with the numerical data at Figure 4.

The behavior near $\mu=0$ is examined next. In Figure 5 we plot $\varepsilon_{0,1}^{ \pm}(n)$, the first and second $\varepsilon$ value at which $\gamma_{\varepsilon, n, \mu=0}(t)$ becomes stable, as a function of $n-1$. It shows that $\varepsilon_{k}^{ \pm}(n) \approx \frac{\beta_{k}^{ \pm}}{n-1}(k=0,1)$ in accordance to (3.29), even though $k$ is not sufficiently large for the asymptotic estimates to hold.

For larger $k$ values, the oscillatory behavior in $\log \varepsilon$ of $\operatorname{Re}\left(\lambda_{n}(\mu=0, \varepsilon)\right)$ is shown in Figures 6 and 7. 

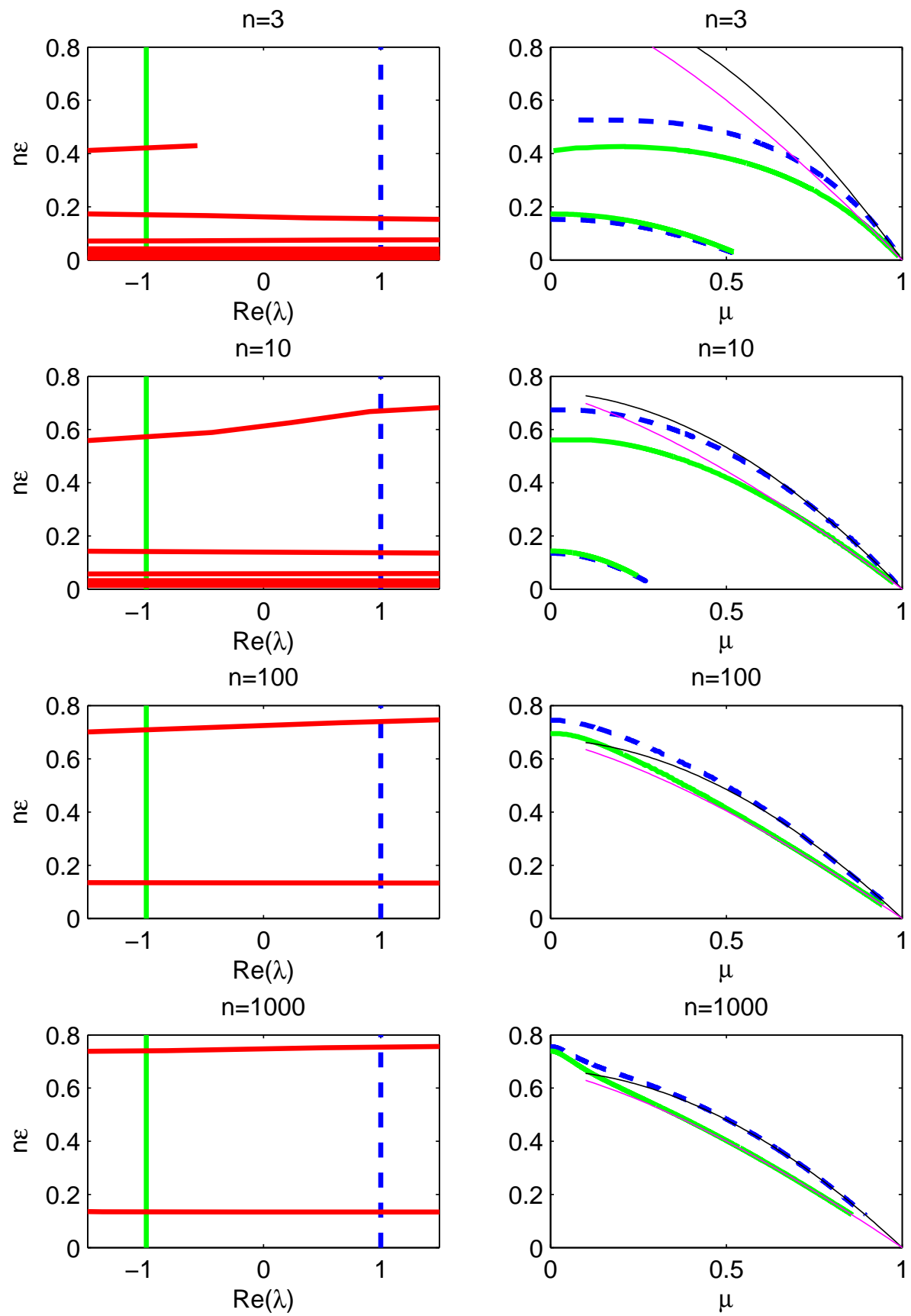

FIGURE 4. Bifurcation diagram for the power-law potential. Left: real part of the eigenvalue $\lambda$ at $\mu=0$. Right: Wedges of stability in $(\mu, n \varepsilon)$ space (note that the $\varepsilon$-axis is scaled with $n$ ). The stability wedges lie between the saddle-center bifurcation curves (dotted lines) and the period doubling bifurcation curves (solid lines). The asymptotic predictions (thin lines) of formulae (3.27),(3.26) for the first wedge are shown. 


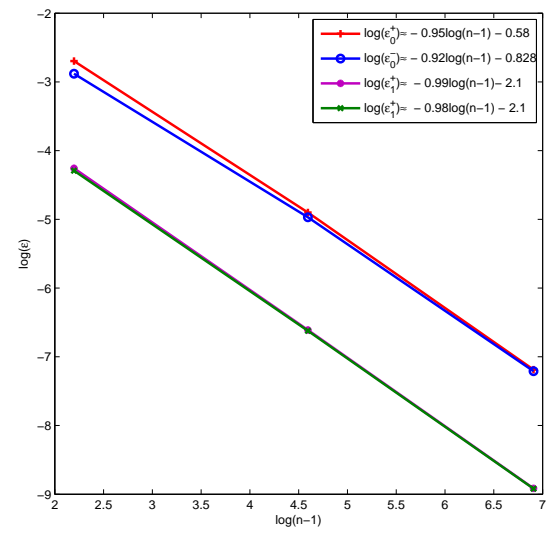

FIGURE 5. The intersection of the first and second wedges of stability with the $\varepsilon$-axis $\left(\varepsilon_{0,1}^{ \pm}(n)\right)$ is shown to scale like $1 / n$.

Indeed, in the proof of Proposition 2, it is established that for the power-law potential, at $\alpha=1$ (see Appendix B):

$$
\operatorname{Tr}(A)=G(0,1)\left(\frac{h(n-1)}{\varepsilon}\right)^{\frac{1}{4}} d\left(1+\frac{d}{R}\right) \sin \left(2 \sqrt{\frac{h}{\varepsilon(n-1)}}+2 \varphi(0,1)\right)+\ldots
$$

with $G(0,1), \varphi(0,1)$ some constants, and thus, using $(3.1), \lambda_{n}(\mu=0, \varepsilon)$ may be estimated in this asymptotic limit; Figure 7 shows the agreement between the numerical computation and the asymptotic form for sufficiently small $\varepsilon$; we fitted $G(0,1)=1.85, \varphi(0,1)=1.1 \pi$ for the $n=3$ case and used these for the $n=10$ case, suggesting that these constants are indeed independent of $n$ as predicted by (4.1).

Figure 6 shows the $\varepsilon^{-\frac{1}{4}}$ envelope of $\operatorname{Re}\left(\lambda_{n}(\mu=0, \varepsilon)\right.$ ) for finite $n$ (similar fitting for the Gaussian case gives rise to a $\varepsilon^{-0.61}$ envelope). In particular, this finding shows that for very small $\varepsilon$ values approaching the cusp limit, the orbit $\gamma_{\varepsilon, n, \mu=0}(t)$ has increasingly large multipliers which grow, on the appropriately defined subsequence of $\varepsilon$ values, as $((n-1) / \varepsilon)^{1 / 4}$.

4.2. Non-linear stability - Phase space plots. To support the claim that for $(\mu, \varepsilon)$ values inside the stability wedges the linearly stable periodic orbit $\gamma_{\varepsilon, \mu}(t)$ is surrounded by island of effective stability (i.e. that KAM tori survive in its neighborhood), we choose parameter values inside the wedges (using Figure 4 right) and integrate the $2 n$ equations of motion directly. The $\left(x_{1}, p_{1}\right)$ projection of the return map to the section $\xi_{s}=\frac{L-R}{2}$ for the power-law potential with $n=10$ is shown in Figure 8 (left column). The islands of effective stability are clearly observed in this projection. To examine the non-degeneracy of these islands to asymmetric perturbations, we introduce the following family of potentials:

$$
V_{k}^{\text {pert }}(x ; \varepsilon)=V_{k}(x ; \varepsilon)+\delta a_{k} V_{k}(x ; \varepsilon),
$$

where $a_{k}$ are uniformly distributed on the unit interval (i.e. we consider the case by which each sphere has a slightly different potential). The phase portraits of the perturbed motion with $\delta=0.001$ are shown in the right column of Figure 8 (we do verify that the projection 

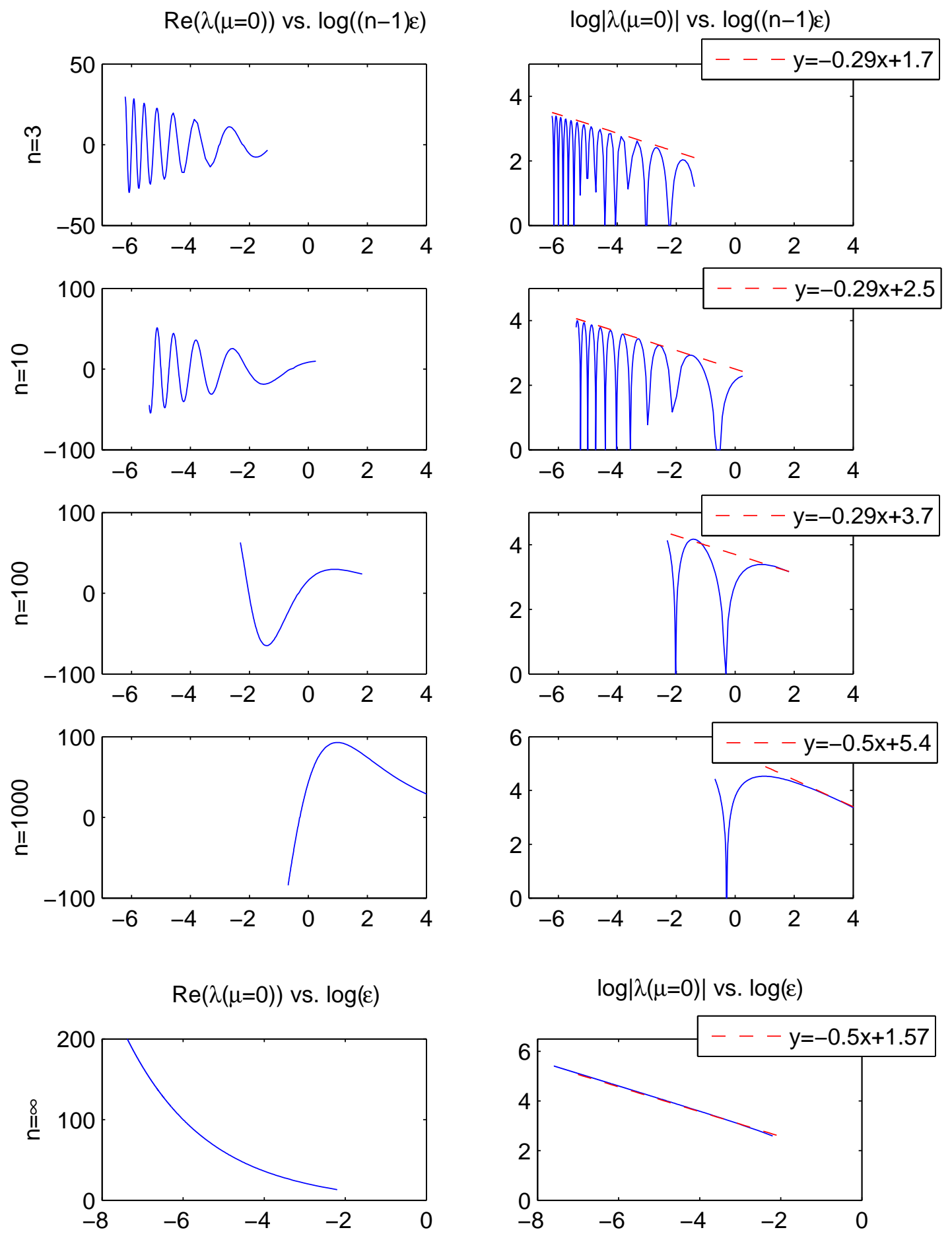

FIGURE 6. The oscillatory nature of the Floquet multipliers at $\mu=0$ for several $n$ values is shown. 

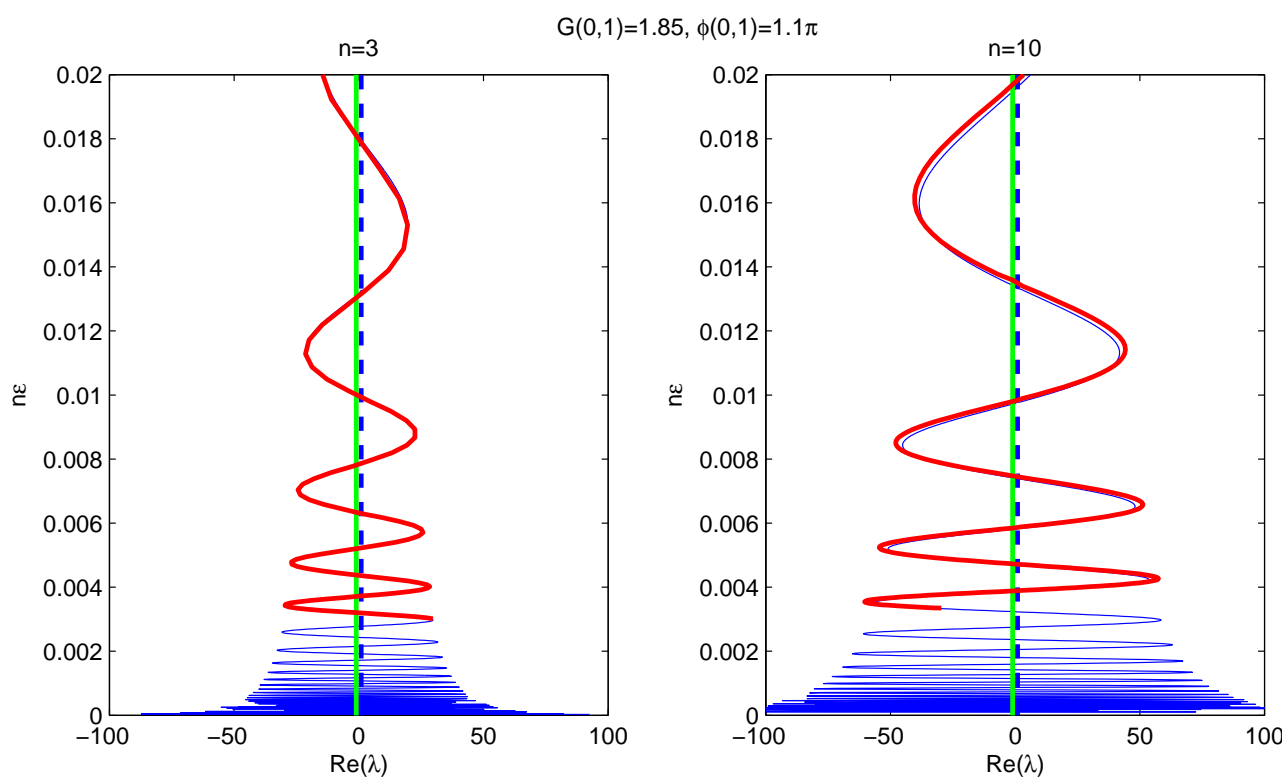

FIGURE 7. Oscillations of $\Re(\lambda(\mu=0 ; \varepsilon))$ at $n=3,10$. Thin line (blue) - analytical estimates (eq. (4.1)). Thick line (red) - numerical computations.

plots of $X=\|x-\gamma(0)\|, P=\|\dot{x}-\dot{\gamma}\|$ remain bounded, namely that there is no instability in any direction of the 20-dimensional phase space).

\section{DisCUSSION}

We have constructed a set of examples that show that for an $n$-dimensional dispersing billiard, for any finite $n$, symmetric corners with $n$ faces can produce islands of effective stability when the billiard is replaced by a more realistic model of a particle moving in a steep repelling potential, for arbitrarily high values of the steepness of the potential. In particular, for a certain symmetric geometry, we have found a specific (diagonal) periodic orbit for which we proved that for any $n$ there is a countable set of wedges in the parameter plane where the periodic orbit is linearly stable. As the steepness parameter, $\varepsilon^{-1}$, tends to infinity, these stability zones do not disappear and remain in a finite region of the parameter plane up to $\varepsilon=0$ that corresponds to the (dispersing) billiard limit. Moreover, we were able to estimate the width and location of these wedges for the power-law potentials. The qualitative results and the asymptotic formulae were supported by numerical computations for the power-law, the Gaussian and the exponential potentials. Finally, we conjecture that for most parameter values in the wedges, where the periodic orbit is linearly stable, a region of effective stability is created (namely, KAM-tori exist, i.e. despite the symmetric form of the potential, the behavior near the elliptic points is similar to the behavior near generic elliptic points). This conjecture is supported by numerical simulations for several $n$ values, for both the power-law and the Gaussian potentials: in these simulations islands of effective stability surviving small symmetry breaking perturbations of the potential are clearly seen (see Figure 8, where projections of islands in 20-dimensional phase space are shown for the power-law potential). 
without perturbation
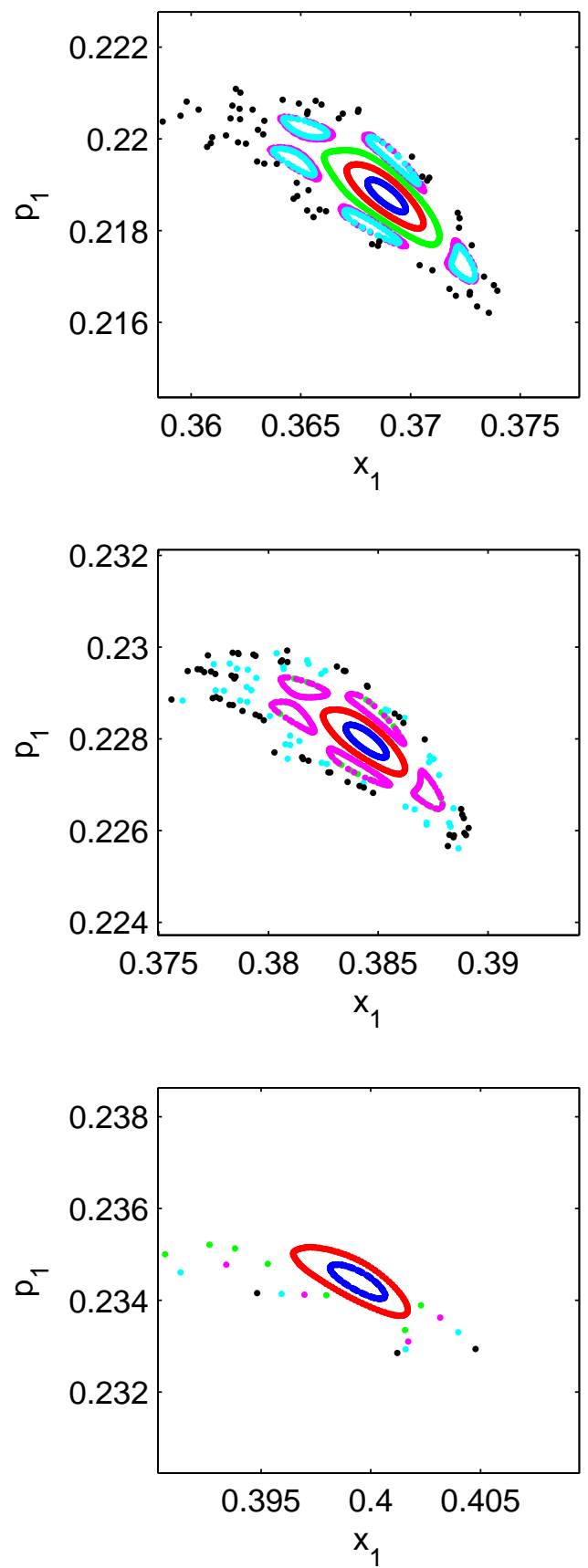

with perturbation $(\delta=0.001)$

$$
\mu=0
$$

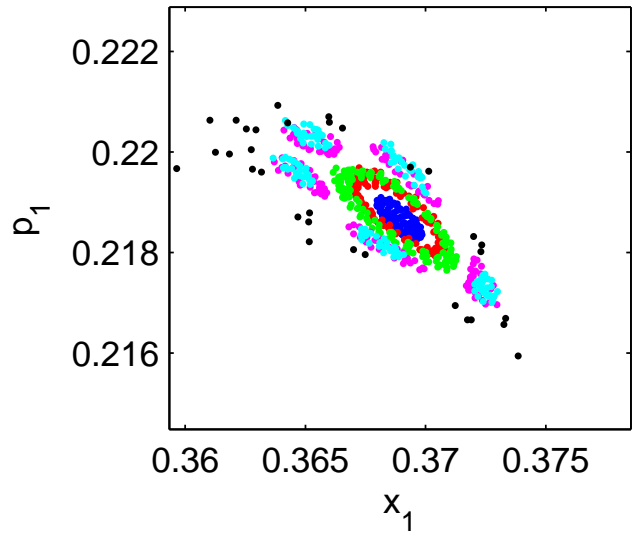

$\mu=0.1$

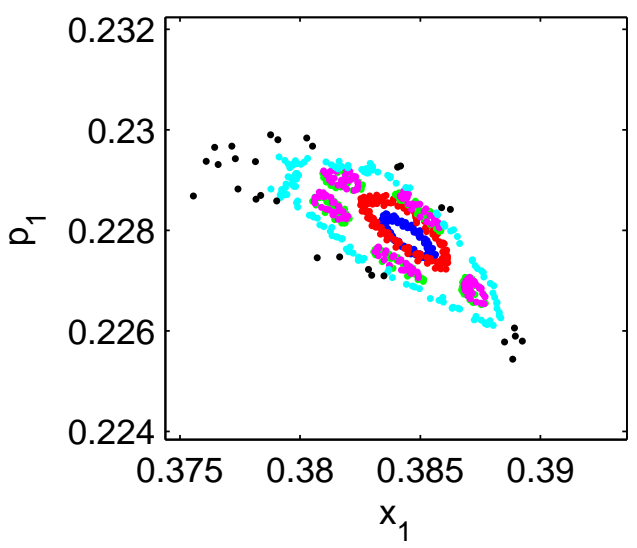

$\mu=0.2$

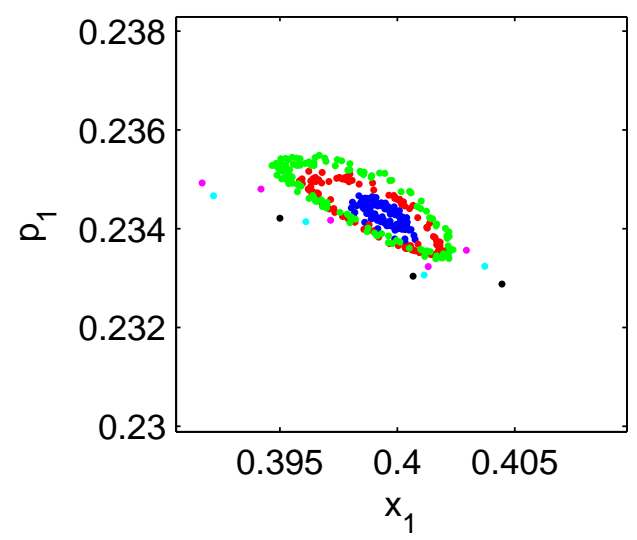

FIGURE 8. Islands in a 20 dimensional symmetric (left) and asymetric(right) systems. Parameter values are chosen inside the first wedge of stability (see figure 4): $\varepsilon=0.0625, \mu=0,0.1,0.2$. Return map projection to the $\left(x_{1}, p_{1}\right)$ plane is shown for the power-law potential with $\alpha=1$. On the right panel the potentials (4.2) with $\delta=0.001$ are used. 
From the mathematical point of view, one generally expects that smooth Hamiltonian systems will have islands of stability. Here, we go beyond genericity type results - we identified specific mechanisms by which the ergodicity and hyperbolicity of the underlying dispersing billiard are destroyed, and a stable motion is created in the problem of a particle moving in a smooth, steep repelling $n$-dimensional potential. The proofs construction includes estimates for the scaling of the stability zones with the control parameters and a description of the bifurcation sequence associated with their creation - such explicit results may be of interest in specific applications.

Admittedly, the presented construction has two limitations which we hope to abolish in future works; the first is the strong symmetry under which the example is constructed; it leads to a highly degenerate spectra - in fact all the non-trivial Floquet multipliers collapse onto only one pair $(\lambda, 1 / \lambda)$, (which is shown to belong to the unit circle in the intervals of stability). Thus, in the symmetric case resonance phenomena must be studied. When the symmetry is slightly broken, either all the eigenvalues remain on the unit circle, or some of them may bifurcate in quadruples to a Hamiltonian Hopf bifurcation. Such possibility may pose difficulties in proving that the periodic orbit remains stable (though one would expect that even in this case stable regions will be created, see $[8,9])$. The other limitations is that the constructed mechanism for the creation of islands requires an $n$-corner - it corresponds to the intersection of $n$ truly $n$-dimensional strictly dispersing scatterers in an $n$-dimensional space. Currently, the most interesting applications of high-dimensional billiards $(n>3)$ are concerned with the problem of $N$ particles in a $d$ dimensional box. In this case the scatterers in the $n=N d$-dimensional configuration space are cylinders with only $d-1$ dispersing directions $[32,27]$, and the phase space structure may prohibit the appearance of the symmetric $n$-corners considered here.

We believe that both of these issues may be resolved in future works. Indeed, the main ingredient in our construction is the concurrent singularity in $n-1$ directions which is induced by the $n$-corner. We conjecture that it is possible to produce islands (non-degenerate elliptic orbits) in any smooth dispersing billiard family in which singular orbits are controlled by $n-1$ independent parameters (here the angles between the $n$ faces of the corner). The symmetric settings are simply convenient for collapsing the number of independent control parameters (here to one). Furthermore, we conjecture that the set of billiards having such singular orbits that produce elliptic islands are dense in the family of Sinai billiards ${ }^{12}$. Hence, while we did not prove yet that a system of $N$ soft particles in $d$-dimensional box is non-ergodic, we can now state that it is likely to be true - if strictly dispersive geometries give rise to elliptic islands, semi-dispersing geometries should do so as well. The methodologies we develop may shed light on the scaling of the non-ergodic components with $N$ and $\varepsilon$, supplying interesting insight on the Boltzmann ergodic hypothesis: while in the hard sphere case Sinai's works show that there is no need to consider the large $N$ limit (which is a major ideological cornerstone in Boltzmann's argument), $N$ does enter into the estimates of the non-ergodic component volume (and possibly their stickiness properties) in the smooth case.

\footnotetext{
${ }^{12}$ The recent results of [4], in which hyperbolicity is proved for finite range potentials that have discontinuous derivatives at their outer perimeter, is consistent with these conjectures - we propose that in that work the hyperbolicity is linked to the lack of smoothness of the potentials.
} 


\section{ACKNOWLEDGEMENT}

We thank Prof. Uzy Smilansky for stimulating discussions. We acknowledge support by the Israel Science Foundation (Grant 926/04), the Minerva foundation and the joint Russian-Israeli grant.

\section{APPENDIX A. LINEARIZED BEHAVIOR NEAR THE CORNER}

Here we construct the linearized reflection matrix near the corner in the finite $\mu$ case and establish Proposition 1 regarding the stability wedge width in this limit. Then we consider the limit of $\mu \rightarrow 0^{+}$: we construct the reflection matrix $C$ in this limit and establish Lemma 6.

First we present the proof of Lemma 4 regarding the form of $C$, the matrix corresponding to the linearized map near the corner: $\left(y(-\Delta t), y^{\prime}(-\Delta t)\right) \mapsto\left(y(\Delta t), y^{\prime}(\Delta t)\right)=$ $C\left(y(\Delta t), y^{\prime}(\Delta t)\right)$ in the limit of small $\varepsilon$ and fixed $\mu>0$ :

Proof. On the time interval $[-\Delta t, \Delta t]$ we scale time $t \rightarrow \delta \cdot \tau$ where

$$
\delta=\varepsilon / \mu \text {. }
$$

Note that $\dot{y}(t)$ then changes to $y^{\prime}(\tau) \delta^{-1}$, hence

$$
C=\left(\begin{array}{cc}
1 & 0 \\
0 & \delta^{-1}
\end{array}\right) \hat{C}\left(\begin{array}{ll}
1 & 0 \\
0 & \delta
\end{array}\right)
$$

where $\hat{C}$ is the matrix of the linear map $\left(\begin{array}{c}y(-\sigma) \\ y^{\prime}(-\sigma)\end{array}\right) \mapsto\left(\begin{array}{c}y(\sigma) \\ y^{\prime}(\sigma)\end{array}\right)=\hat{C}\left(\begin{array}{c}y(-\sigma) \\ y^{\prime}(-\sigma)\end{array}\right)$ defined by the rescaled equation (3.2):

$$
y^{\prime \prime}+\delta^{2} a(\tau \delta) y=0
$$

on the interval $\tau \in[-\sigma, \sigma]$, where we denote

$$
\sigma=\Delta t / \delta
$$

Note that $\sigma$ tends to $+\infty$ as $o\left(\varepsilon^{-1}\right)$, because we assume that $\Delta t=o(1)_{\varepsilon \rightarrow 0}$. Let us introduce a new variable $z$ by the rule

$$
\sqrt{1+2 \mu \nu+v^{2}}=1+\varepsilon z
$$

i.e. $z$ is a rescaled distance to the corner. Recall that we choose our parametrization of time along $\gamma$ in such a way that $t=0$ corresponds to the point nearest to the corner. Hence, we have from (2.13),(2.14),(A.5),(2.7) that

$$
\frac{h}{2}=V(z)+O\left(\varepsilon^{\alpha}\right),
$$

i.e. $z(0)$ stays uniformly bounded for all $\varepsilon$. As the velocity $\dot{v}$ is bounded from above by virtue of (2.13),(2.6), it follows that $v(t)-v(0)=O(\Delta t)$ at $|t| \leq \Delta t$, so $z(t)-z(0)=$ $O(\Delta t / \varepsilon)$, i.e. $z=o\left(\varepsilon^{-1}\right)$ for all $t$ from this interval.

It is easy to see that equation (A.3) (see also (3.3)) takes the following form after the rescaling:

$$
y^{\prime \prime}+\left(\frac{1-\mu^{2}}{(n-1) \mu^{2}} V^{\prime \prime}(z)+\varepsilon \tilde{a}(z, \varepsilon)\right) y=0
$$

where $\tilde{a}$ is uniformly bounded and

$$
\tilde{a}=O\left(|z|^{-1-\alpha}\right)
$$


uniformly for all $z$ such that $\varepsilon z$ is small. Equation (2.13) changes to

$$
\frac{h}{2}=\frac{\left(z^{\prime}\right)^{2}}{2}(1+\phi(z, \varepsilon))+V(z)+\tilde{V}(z, \varepsilon)
$$

where

$$
\tilde{V}=O\left(\varepsilon^{\alpha}\right), \text { and } \phi=o(1)_{\varepsilon \rightarrow 0} .
$$

As we mentioned, we consider equations (A.6),(A.8) at $z \leq z^{*}$ with some $z^{*}=o\left(\varepsilon^{-1}\right)$. Therefore, at $z>z^{*}$ we may define $\phi$ and $\tilde{V}$ in an arbitrary way, and we define there $\phi(z)=\phi\left(z^{*}\right)$ and $\tilde{V}(z)=\tilde{V}\left(z^{*}\right)\left(z^{*} / z\right)^{\alpha}$. Then, by virtue of (2.7),(A.9), the potential in the right-hand side of (A.8) uniformly (for all small $\varepsilon$ ) tends to zero as $z \rightarrow+\infty$. Hence, uniformly for all small $\varepsilon$,

$$
z(\tau)=\tau(\sqrt{h}+o(1)) \text { as } \tau \rightarrow \pm \infty .
$$

By plugging this into (A.6), and defining $\tilde{a}(z)=\tilde{a}\left(z^{*}\right)\left(z^{*} / z\right)^{2+\alpha}$ we see from (2.7),(A.7) that equation (A.6) has the form

(A.10) $y^{\prime \prime}+Q(\tau, \varepsilon) y=0 \quad$ where, uniformly for all $\varepsilon, Q=O\left(|\tau|^{-2-\alpha}\right)$ as $\tau \rightarrow+\infty$.

Moreover, $Q$ is continuous with respect to $\varepsilon$ and has a limit (uniformly for all $z$ ) as $\varepsilon \rightarrow 0$ : the limit system is

$$
\begin{gathered}
y^{\prime \prime}+\beta V^{\prime \prime}(z) y=0, \\
\beta=\frac{1-\mu^{2}}{(n-1) \mu^{2}}
\end{gathered}
$$

where $z(\tau)$ solves

$$
\frac{h}{2}=\frac{\left(z^{\prime}\right)^{2}}{2}+V(z)
$$

It is a routine fact that every solution $y(\tau)$ of equation of type (A.10) grows at most linearly as $\tau \rightarrow \pm \infty$; and that there exists a limit for the derivative $y^{\prime}$ :

$$
y^{\prime}(\tau)=D_{1}^{ \pm}+O\left(|\tau|^{-\alpha}\right)
$$

uniformly for any bounded set of initial conditions and for all small $\varepsilon$. Moreover, the solution is bounded as $\tau \rightarrow+\infty$ if and only if $D_{1}^{+}=0$; and the solution stays bounded as $\tau \rightarrow-\infty$ if and only if $D_{1}^{-}=0$. Among the solutions bounded as $\tau \rightarrow+\infty$, there exists exactly one solution $y_{+}$which tends to 1 . Analogously, there exists exactly one solution $y_{-}$which tends to 1 as $\tau \rightarrow-\infty$ :

$$
y_{ \pm}(\tau)=1+O\left(|\tau|^{-\alpha}\right), \quad y_{ \pm}^{\prime}(\tau)=O\left(|\tau|^{-1-\alpha}\right) .
$$

We also take a pair $\hat{y}_{+}(\tau)$ and $\hat{y}_{-}(\tau)$ of solutions such that

$$
\hat{y}_{-}^{\prime}(-\infty)=1, \quad \hat{y}_{+}^{\prime}(+\infty)=1,
$$

hence

$$
\hat{y}_{ \pm}=\tau+O\left(|\tau|^{1-\alpha}\right)
$$

The solutions $\hat{y}_{ \pm}$are not uniquely defined, therefore we now fix a certain canonical choice of them, in order to ensure that they will depend continuously on $\varepsilon$ and other parameters of the problem. To do that, let $\varphi(\tau)$ denote the solution of (A.10) with initial conditions $\varphi(0)=1, \varphi^{\prime}(0)=0$, and let $\psi(\tau)$ be the solution with initial conditions $\psi(0)=0, \psi^{\prime}(0)=1$ (we deal with time-reversible equations, and in this setting $\varphi$ and $\psi$ are, respectively, the 
even and odd solutions of (A.10); we do not use this in the proof of this theorem). Recall that

$$
\operatorname{det}\left(\begin{array}{cc}
\varphi & \psi \\
\varphi^{\prime} & \psi^{\prime}
\end{array}\right)=1
$$

for all $\tau$, by Wronsky formula. As $y_{+}$is defined uniquely (by condition (A.14)), there exist uniquely defined constants $K_{1}$ and $K_{2}$ such that

$$
y_{+}=K_{1} \varphi-K_{2} \psi
$$

(one can show that $K_{1}=\psi^{\prime}(+\infty)$ and $K_{2}=\varphi^{\prime}(+\infty)$, but we do not use this information). We will choose

$$
\hat{y}_{+}=\frac{K_{2}}{K_{1}^{2}+K_{2}^{2}} \varphi+\frac{K_{1}}{K_{1}^{2}+K_{2}^{2}} \psi \text {. }
$$

Note that $\left(y_{+}, \hat{y}_{+}\right)$are related to $(\varphi, \psi)$ by a linear transformation with the determinant equal to 1 . Therefore, by virtue of (A.17),

$$
\operatorname{det}\left(\begin{array}{cc}
y_{+} & \hat{y}_{+} \\
y_{+}^{\prime} & \hat{y}_{+}^{\prime}
\end{array}\right)=1
$$

for all $\tau$. By taking a limit as $\tau \rightarrow+\infty$, we obtain from this formula (see also (A.14),(A.13)) that $\hat{y}_{+}^{\prime}(+\infty)=1$, i.e. thus defined $\hat{y}$ satisfies (A.15),(A.16), as required. Analogously one can fix the choice of $\hat{y}^{-}$; note that

$$
\operatorname{det}\left(\begin{array}{ll}
y_{-} & \hat{y}_{-} \\
y_{-}^{\prime} & \hat{y}_{-}^{\prime}
\end{array}\right)=1 \text {. }
$$

As $y_{+}$and $\hat{y}_{+}$are linearly independent, every solution is a linear combination of them:

$$
y(\tau)=D_{0}^{+} y_{+}+D_{1}^{+} \hat{y}_{+} .
$$

The same solution can be written as

$$
y(\tau)=D_{0}^{-} y_{-}+D_{1}^{-} \hat{y}_{-} .
$$

It follows that the solutions of (A.3) define a continuously depending on $\varepsilon$ scattering matrix $S(\varepsilon)$ :

$$
\left(\begin{array}{c}
D_{0}^{+} \\
D_{1}^{+}
\end{array}\right)=S\left(\begin{array}{c}
D_{0}^{-} \\
D_{1}^{-}
\end{array}\right)
$$

Moreover, the matrix $\hat{C}$ of the map $\left(\begin{array}{c}y(-\sigma) \\ y^{\prime}(-\sigma)\end{array}\right) \mapsto\left(\begin{array}{c}y(\sigma) \\ y^{\prime}(\sigma)\end{array}\right)$ is given by

$$
\hat{C}=\left(\begin{array}{ll}
y_{+}(\sigma) & \hat{y}_{+}(\sigma) \\
y_{+}^{\prime}(\sigma) & \hat{y}_{+}^{\prime}(\sigma)
\end{array}\right) \cdot S(\varepsilon) \cdot\left(\begin{array}{cc}
\hat{y}_{-}^{\prime}(-\sigma) & -\hat{y}_{-}(-\sigma) \\
-y_{-}^{\prime}(-\sigma) & y_{-}(-\sigma)
\end{array}\right) .
$$

Recall that $\sigma \rightarrow+\infty$. By (A.2),(A.25),(A.14),(A.16),(A.13),

$$
C=\left(\begin{array}{cc}
s_{11}+o(1)+\sigma s_{21}(1+o(1)) & s_{21} O\left(\delta \sigma^{2}\right)+O(\delta \sigma) \\
\frac{1}{\delta}\left(s_{21}(1+o(1))+O\left(\sigma^{-1-\alpha}\right)\right) & s_{22}+o(1)+\sigma s_{21}(1+o(1))
\end{array}\right) .
$$

where $s_{i j}(\varepsilon)$ are the entries of the scattering matrix.

The proof of Proposition 1, regarding the width of the stability wedges for small $\beta$ values is established next: 
Proof. The stability zone corresponds to $|\operatorname{Tr}(A)|<2$. By (3.14),(A.1) the boundary $\operatorname{Tr}(A)=$ 2 is given by

$$
\varepsilon\left(2-\left(s_{11}+s_{22}\right)\left(1+\frac{2 d}{R}\right)+o(1)_{\varepsilon \rightarrow 0}\right)=\frac{2}{\sqrt{h}} d\left(1+\frac{d}{R}\right) s_{21},
$$

and the boundary $\operatorname{Tr}(A)=-2$ is given by

$$
\varepsilon\left(-2-\left(s_{11}+s_{22}\right)\left(1+\frac{2 d}{R}\right)+o(1)_{\varepsilon \rightarrow 0}\right)=\frac{2}{\sqrt{h}} d\left(1+\frac{d}{R}\right) s_{21},
$$

where $s_{i j}$ are the entries of the scattering matrix $S(\mu, \varepsilon)$ of equation (A.6). At $\beta=\frac{1-\mu^{2}}{(n-1) \mu^{2}}=$ $0, \varepsilon=0$ equation (A.6) (the finite $\varepsilon$ version of (3.9)) degenerates into $y^{\prime \prime}=0$, and the scattering matrix is equal to the identity. Thus, at $\beta$ close to 0 and small $\varepsilon$, we find that

$$
s_{11}+s_{22}=2+o(1)
$$

and

$$
s_{21}=q_{1} \beta+q_{2} \varepsilon+o(|\varepsilon|+|\beta|)
$$

where

$$
q_{1}=\left.\frac{\partial s_{21}}{\partial \beta}\right|_{(\beta=0, \varepsilon=0)}
$$

and

$$
q_{2}=\left.\frac{\partial s_{21}}{\partial \varepsilon}\right|_{(\beta=0, \varepsilon=0)} .
$$

(while $S$ may be non differentiable in $\varepsilon$ for general $\beta$, it can be shown, using (A.6), that at $\beta=\varepsilon=0$ the expansion (A.30) is valid). Thus, by plugging (A.29),(A.30),(3.23) into (A.27),(A.28), we find the following equations for the boundaries of the stability zone near $(\beta=0, \varepsilon=0)$ :

$$
\varepsilon\left(\frac{2 \sqrt{h}}{d+R}+q_{2}\right)+o(\varepsilon)=-\sqrt{h} I \beta+o(\beta)
$$

and

$$
\varepsilon\left(\frac{2 \sqrt{h}}{d}+q_{2}\right)+o(\varepsilon)=-\sqrt{h} I \beta+o(\beta) .
$$

As we see, in order to prove the lemma, it remains to show that

$$
q_{2}=2 \sqrt{h}
$$

By definition, $s_{21}$ equals to $y_{-}^{\prime}(+\infty)$ where $y_{-}(\tau)$ is the solution of (A.6) that satisfies $y_{-}(-\infty)=1$. Let us write (A.6) in the form (A.10). By differentiating (A.10) we find that the derivative $u(\tau)=\frac{\partial}{\partial \varepsilon} y_{-}(\tau)$ satisfies

$$
u^{\prime \prime}+Q u=-\frac{\partial Q}{\partial \varepsilon} y_{-} .
$$

As $Q=0$ and $y_{-}=1$ for all $\tau$ at $\beta=0, \varepsilon=0$, we obtain that

$$
q_{2}=\left.u^{\prime}(+\infty)\right|_{(\beta=0, \varepsilon=0)}=-\int_{-\infty}^{+\infty} \frac{\partial Q}{\partial \varepsilon} d \tau
$$

From (A.6),(A.3),(3.3), we find

$$
\left.\frac{\partial Q}{\partial \varepsilon}\right|_{(\beta=0, \varepsilon=0)}=\left.\tilde{a}(z, 0)\right|_{\beta=0}=V^{\prime}(z) .
$$


This gives us (see also (3.11))

$$
\left.q_{2}=-\int_{-\infty}^{+\infty} V^{\prime}(z(\tau)) d \tau=\int_{-\infty}^{+\infty} z^{\prime \prime}(\tau)\right) d \tau=z^{\prime}(+\infty)-z^{\prime}(-\infty)=2 \sqrt{h}
$$

as required.

Next we find the form of $C$ in the limit at which both $\mu$ and $\varepsilon$ are small. Here, as in Lemma 4 , on the time interval $[-\Delta t, \Delta t]$ we scale time $t \rightarrow \delta \cdot \tau$, yet here we choose a different scaling coefficient $\delta$ (compare with (A.1)):

$$
\delta=\frac{\varepsilon}{\sqrt{2 \varepsilon+\mu^{2}}} .
$$

Obviously, $\delta \rightarrow 0$ (at least as $O(\sqrt{\varepsilon})$ ) as $\varepsilon$ and $\mu$ tend to zero. Then, the matrix $C$ is given by formula (A.2), where $\hat{C}$ is the corresponding matrix for system (A.3) obtained from (3.2) by the new time-scaling.

With such scaling, system (A.3) gets the form

$$
y^{\prime \prime}+\frac{1}{2 \varepsilon+\mu^{2}}\left(\frac{1-\mu^{2}}{n-1} V^{\prime \prime}(z)+\varepsilon \tilde{a}(z, \varepsilon)\right) y=0
$$

where $\tilde{a}$ is uniformly bounded and satisfies (A.7) for all $z$ such that $\varepsilon z$ is small. The equation for $z(\tau)$ changes from (A.8) to

$$
\frac{\left(z^{\prime}\right)^{2}}{2}(1+\varepsilon z)^{2}=\left(\frac{h}{2}-V(z)-\tilde{V}(z, \varepsilon)\right)(M+(1-M) z(1+\varepsilon z / 2)),
$$

where

$$
M(\varepsilon, \mu)=\frac{\mu^{2}}{\mu^{2}+2 \varepsilon},
$$

and $\tilde{V}$ satisfies (A.9). Like in the proof of Lemma 4, we consider only the interval $z \leq z^{*}$ with $z^{*}=o\left(\varepsilon^{-1}\right)$, so outside this interval we may replace the terms $\varepsilon z$ with $\varepsilon z^{*}$ both in the right- and left-hand side of (A.34), and replace $\tilde{V}(z, \varepsilon)$ with $\tilde{V}\left(z^{*}, \varepsilon\right)\left(z^{*} / z\right)^{\alpha}$. Then $z(\tau)$ tends to $+\infty$ linearly with $\tau$ or faster, with the velocity bounded away from zero. It follows that like in of Lemma 4, the system (A.33),(A.34) belongs to the class (A.10), hence the matrix $\hat{C}$ is expressed by formula (A.25) via the scattering matrix $S(\varepsilon, \mu)$ defined by (A.24).

Lemma 7. For small $\Delta t$ and sufficiently small $\mu$ and $\varepsilon$, the linearized map about the diagonal orbit near the corner $C:\left(y(-\Delta t), y^{\prime}(-\Delta t)\right) \mapsto\left(y(\Delta t), y^{\prime}(\Delta t)\right)=C\left(y(\Delta t), y^{\prime}(\Delta t)\right)$ is of the form

$$
C=\frac{K_{1}^{2}-K_{2}^{2}}{K_{1}^{2}+K_{2}^{2}}\left(\begin{array}{cc}
1+O\left((\delta / \Delta t)^{\alpha}\right) & O(\Delta t) \\
O\left(\delta^{\alpha}(\Delta t)^{1+\alpha}\right) & 1+O\left((\delta / \Delta t)^{\alpha}\right)
\end{array}\right)+K_{1} K_{2} \tilde{C},
$$

where $\delta \rightarrow 0$ as $\varepsilon \rightarrow 0$, $\tilde{C}$ is a matrix whose exact form is irrelevant here and $K_{1}, K_{2}$ are the coefficients of the even and odd components of the solution $y_{-}(\tau)$ of equation (A.33) with z solving (A.34).

Proof. Here we will use the time-reversibility of equation (A.33),(A.34): if $y(\tau)$ is its solution, then $y(-\tau)$ is a solution as well. It follows that

$$
y_{-}(\tau)=y_{+}(-\tau)
$$

hence, by (A.18),

$$
y_{-}(\tau)=K_{1} \varphi(\tau)+K_{2} \psi(\tau)
$$


where $\varphi$ and $\psi$ are, respectively, the even and odd solutions of (A.33). Then, analogously to (A.19),

$$
\hat{y}_{+}=-\frac{K_{2}}{K_{1}^{2}+K_{2}^{2}} \varphi+\frac{K_{1}}{K_{1}^{2}+K_{2}^{2}} \psi .
$$

From (A.18),(A.19),(A.37),(A.38),(A.22),(A.23),(A.24) we obtain the following formula for the scattering matrix:

$$
S=\left(\begin{array}{cc}
\frac{K_{1}^{2}-K_{2}^{2}}{K_{1}^{2}+K_{2}^{2}} & -\frac{2 K_{1} K_{2}}{\left(K_{1}^{2}+K_{2}^{2}\right)^{2}} \\
2 K_{1} K_{2} & \frac{K_{1}^{2}-K_{2}^{2}}{K_{1}^{2}+K_{2}^{2}}
\end{array}\right) .
$$

By (A.2), (A.25), (A.14), (A.16), (A.13), (A.4), (A.39) the required form of $C$, namely (A.36) is found.

Finally we establish Lemma 6 regarding the stability wedges in this limit:

Proof. As before, we represent the monodromy matrix $A$ as the product of the two matrices $B$ and $C$. Since $B$ corresponds to the regular part of the diagonal orbit and is independent of $\mu$, Lemma 3 applies in this small $\mu$ and $\varepsilon$ limit as well and the matrix $B$ is given by (3.7). As $\delta$ and $\Delta t$ tend to zero, while $\Delta t$ does this sufficiently slowly, we find from (A.36),(3.7) that

$$
\operatorname{Tr}(A)= \begin{cases}-2\left(1+\frac{2 d}{R}\right)+o(1)_{(\varepsilon, \mu) \rightarrow 0}<-2 & \text { at } K_{1}=0 \\ 2\left(1+\frac{2 d}{R}\right)+o(1)_{(\varepsilon, \mu) \rightarrow 0}>2 & \text { at } K_{2}=0\end{cases}
$$

The sought stability intervals on the curve $\mathcal{L}$ correspond to $|\operatorname{Tr}(A)|<2$. Therefore, by virtue of (A.40), we will prove the lemma if we show that there exist a converging to zero sequence of values of $(\varepsilon, \mu) \in \mathcal{L}$ which correspond to $K_{1}=0$ and a converging to zero sequence of values of $(\varepsilon, \mu) \in \mathcal{L}$ for which $K_{2}=0$.

By (A.39), vanishing of $K_{1}$ or $K_{2}$ corresponds to vanishing of $s_{21}$, i.e. to the boundedness of the solution $y_{-}$of (A.33). At $K_{1}=0$ we have from (A.37) that $y_{-}=\psi$, i.e. the bounded solution is odd, while at $K_{2}=0$ the bounded solution $y_{-}=\varphi$ is even. Thus, $K_{1}=0$ corresponds to the existence of a bounded solution $y_{-}$with an odd number of zeros, and $K_{2}=0$ corresponds to the existence of a bounded solution with an even number of zeros. It remains to note that the coefficient $\frac{1-\mu^{2}}{(n-1)\left(2 \varepsilon+\mu^{2}\right)}$ of $V^{\prime \prime}(z) y$ in (A.33) tends to $+\infty$ as $(\varepsilon, \mu) \rightarrow 0$. From that, exactly like in the proof of Theorem 2, we obtain that the number of zeros of $y_{-}$tends to infinity as $(\varepsilon, \mu) \rightarrow 0$. We also showed in the proof of Theorem 2 that each time the number of zeros changes, the increase is exactly 1 . Now, the required existence of a converging to zero sequence of values of $(\varepsilon, \mu) \in \mathcal{L}$ which correspond to the existence of a bounded solution with odd number of zeros (i.e. $K_{1}=0$ ) and a converging to zero sequence of values of $(\varepsilon, \mu) \in \mathcal{L}$ which correspond to the existence of a bounded solution with even number of zeros $\left(K_{2}=0\right)$ follows immediately. 


\section{APPENDIX B. THE POWER-LAW POTENTIAL}

To establish Proposition 2, we integrate equation (A.33) with the power-law potential in the asymptotic limit of small $(\varepsilon, \mu)$. In fact, we show below that by parameterizing the $(\mu, \varepsilon)$ plane by the parameters

$$
(\rho, M)=\left(\sqrt{2 \varepsilon+\mu^{2}}, \frac{\mu^{2}}{2 \varepsilon+\mu^{2}}\right)
$$

we obtain estimates to the width of the wedge for all sufficiently small $\rho$ uniformly in $M$. We first introduce some notations. Recall that the parabolas emanating from the origin $\mathcal{L}_{M}=\left\{(\mu, \varepsilon): 2 \varepsilon M=\mu^{2}(1-M)\right\}$ were defined for a fixed parameter $M \in[0,1]$ and that $\rho$ is used to parameterize these curves. Let

$$
J(M)=\int_{(2 / h)^{1 / \alpha}}^{+\infty} \frac{d z}{z \sqrt{\left(h z^{\alpha}-2\right)(M+(1-M) z)}} .
$$

In particular,

$$
J(1)=\frac{\pi}{\sqrt{2} \alpha} \text { and } J(0)=\left(\frac{h}{2}\right)^{1 / 2 \alpha} \frac{\sqrt{2}}{\alpha} \int_{0}^{\pi / 2}(\sin \theta)^{1 / \alpha} d \theta
$$

and at $\alpha=1, J(0)=\sqrt{h}$. Let

$$
P(\rho, M)=\sqrt{M h}\left(\kappa^{\alpha} \rho^{2} \frac{n-1}{\alpha(\alpha+1)}\right)^{\frac{1}{\alpha+2}},
$$

where $\kappa=\kappa(\rho, M)$ solves the equation

$$
\left(h \frac{1-M}{4} \kappa^{1+\frac{\alpha}{\alpha+2}}+\sqrt{M h} \kappa^{\frac{\alpha}{\alpha+2}}\right)^{\alpha+2} \rho^{2}=\frac{\alpha(\alpha+1)}{n-1} .
$$

Note that $\kappa \rightarrow+\infty$ as $\rho \rightarrow 0$, while $P(\rho, M)$ remains bounded: $P \in[0,1]$. Moreover, one can rewrite (B.5) in the following form (recall that $\delta=\frac{\varepsilon}{\rho}$ and $\sqrt{M}=\frac{\mu}{\rho}$, see (A.32),(A.35)):

$$
\frac{h}{2} \kappa \delta+\mu \sqrt{h}=(\rho / \kappa)^{\alpha /(\alpha+2)}\left(\frac{\alpha(\alpha+1)}{n-1}\right)^{1 /(\alpha+2)},
$$

from which it follows immediately that

$$
\kappa \delta=\kappa \frac{\rho(1-M)}{2}=o(1) .
$$

Consider an equation

$$
y^{\prime \prime}(\theta)+\frac{1}{\left((1-P) \theta^{2}+P \theta\right)^{\alpha+2}} y(\theta)=0
$$

defined at $\theta>0$. In the limit $\theta \rightarrow+0$, the coefficient of $y$ in (B.8) tends to $+\infty$, which produces fast oscillations in $y$ : every solution has the asymptotic given by

$$
y(\theta) \approx E_{1}\left((1-P) \theta^{2}+P \theta\right)^{\frac{\alpha+2}{4}} \cos \left(\int_{\theta}^{+\infty} \frac{d \theta}{\left((1-P) \theta^{2}+P \theta\right)^{1+\alpha / 2}}\right)-
$$

$$
E_{2}\left((1-P) \theta^{2}+P \theta\right)^{\frac{\alpha+2}{4}} \sin \left(\int_{\theta}^{+\infty} \frac{d \theta}{\left((1-P) \theta^{2}+P \theta\right)^{1+\alpha / 2}}\right)
$$


with some constant $E_{1,2}$. The asymptotic behavior as $\theta \rightarrow+\infty$ is given by (A.22),(A.16),(A.14),(A.20) i.e.

$$
y(\theta)=F_{0}\left(1+O\left(\theta^{-\alpha}\right)\right)+F_{1} \theta\left(1+O\left(\theta^{-\alpha}\right)\right)
$$

with some constant $F_{0,1}$. Thus, solutions of (B.8) define the scattering matrix $\hat{S}(P, \alpha)$ :

$$
\left(\begin{array}{c}
F_{0} \\
F_{1}
\end{array}\right)=\hat{S}\left(\begin{array}{c}
E_{1} \\
E_{2}
\end{array}\right) \text {. }
$$

For convenience of later computation we use the following general form for $\hat{S}$ :

$$
\hat{S}(P, \alpha)=\left(\begin{array}{cc}
\sqrt{g} \cos \zeta & \sqrt{g} \sin \zeta \\
\sqrt{G} \cos \varphi & \sqrt{G} \sin \varphi
\end{array}\right) .
$$

Notice that $\operatorname{det} \hat{S}=1$ by construction, hence

$$
\sqrt{G g} \sin (\varphi-\zeta)=1 .
$$

where $G, g, \varphi, \zeta$ depend only on $P$ and $\alpha$.

Proposition 3. In the case of the power-law potential $V(Q, \varepsilon)=\left(\frac{\varepsilon}{Q}\right)^{\alpha}$, every curve $\mathcal{L}_{M}$, $M \in[0,1]$, intersects infinitely many stability tongues; the intersections happen near (see (B.2))

$$
\rho=\rho_{k}=\frac{2 J(\alpha, M) \sqrt{\alpha(\alpha+1)}}{\pi k \sqrt{n-1}},
$$

and the length of the intervals is given by (see (B.4),(B.5) and (B.7))

$$
(\Delta \rho)_{k} \approx \frac{\rho_{k}}{\pi k} \frac{\sqrt{h}}{G\left(P\left(\rho_{k}, M\right), \alpha\right) d\left(1+\frac{d}{R}\right)} \rho_{k} \kappa\left(\rho_{k}, M\right)(1-M) .
$$

Proof. As before, we need to estimate the scattering matrix $S$ for the rescaled equation (A.33). For the power-law potential we have $V^{\prime \prime}>0$, so the coefficient of $y$ is positive at small $\varepsilon$ for all $z$. Thus, we may represent equation (A.33) in the form

$$
y^{\prime \prime}+\Omega^{2}(\tau, \varepsilon, \mu) y=0,
$$

We consider equation (B.16) separately on the interval $|\tau| \leq R$, and on the intervals $|\tau|>R$, where $R(\varepsilon, \mu)$ tends sufficiently slowly to infinity as $(\varepsilon, \mu) \rightarrow 0$ (i.e. as $\rho \rightarrow 0$ ). $R$ is chosen so that for $|\tau| \leq R$ the frequency $\Omega$ is large, hence $y$ is highly oscillatory, and so its envelope is found below by the method of averaging. Then, we show that on the intervals $|\tau|>R$ (B.16) limits, after some rescaling, to (B.8). Thus, the scattering matrix of (A.33) is found by composing the rescaled $\hat{S}$ with the oscillatory solution envelope and then with the rescaled $\hat{S}^{-1}$. Once $S$ is found, the stability regions are found from trace $A$.

Let $R(\varepsilon, \mu)$ be chosen such that $\Omega$ tends to $+\infty$ uniformly on the interval $|\tau| \leq R$, as $(\varepsilon, \mu) \rightarrow+0$ (it tends to $+\infty$ indeed on any finite interval of $\tau$ - hence it tends to $+\infty$ on any sufficiently slowly growing interval as well). Then, there exists a limit of (A.33) and (A.34) by which

$$
\lim _{\rho \rightarrow 0} \rho^{2} \Omega^{2}=\frac{\alpha(\alpha+1)}{(n-1) z^{\alpha+2}},
$$

with $z(\tau)$ solving

$$
\left(\frac{h}{2}-\frac{1}{z^{\alpha}}\right)(M+(1-M) z)=\frac{\left(z^{\prime}\right)^{2}}{2}, \quad z^{\prime}(0)=0 .
$$


Let us apply an averaging procedure to (B.16) on the interval $\tau \in[-R, R]$ : define $(r, \phi)$ by

$$
\sqrt{\Omega} y=\sqrt{r} \cos \phi, \quad \frac{1}{\sqrt{\Omega}} y^{\prime}=\sqrt{r} \sin \phi .
$$

Then, equation (B.16) takes the form

or, after we introduce the fast and slow phases

$$
r^{\prime}=\frac{\Omega^{\prime}(\tau)}{\Omega} r \cos 2 \phi, \quad \phi^{\prime}=-\Omega-\frac{\Omega^{\prime}(\tau)}{2 \Omega} \sin 2 \phi,
$$

$$
\eta=\int \Omega(\tau) d \tau, \quad \Phi=\phi+\eta
$$

the following form

$$
\frac{d r}{d \eta}=\omega r \cos 2(\Phi-\eta), \quad \frac{d \Phi}{d \eta}=-\frac{\omega}{2} \sin 2(\Phi-\eta)
$$

where $\omega:=\Omega^{\prime}(\tau) / \Omega^{2}(\tau)$; by (B.17) (and since $z^{\prime}$ is bounded by (B.18))

$$
\omega=O\left(\sqrt{2 \varepsilon+\mu^{2}}\right)
$$

uniformly for $|\tau| \leq R$ (provided $R$ grows sufficiently slowly). Since $\omega$ in (B.20) is small, by virtue of the averaging principle, the solutions of (B.20) are close to the solutions of the averaged (with respect to $\eta$ ) system for every $o\left(\omega^{-2}\right)$-long interval of values of $\eta$.

In fact, the total change in $\eta$ cannot exceed $\int_{-\infty}^{+\infty} \Omega(\tau) d \tau=O\left(\rho^{-1}\right)=o\left(\omega^{-2}\right)$ (see (B.19),(B.17),(B.21)). Hence, for all $\tau \in[-R, R]$, the solutions of (B.20) remain close to the solutions of the system averaged with respect to $\eta$, which is simply

$$
\frac{d r}{d \eta}=0, \quad \frac{d \Phi}{d \eta}=0
$$

Thus, the evolution from $\tau=-R$ to $\tau=R$ is, to the leading order, just a rotation by the angle $-\int_{-R}^{R} \Omega(\tau) d \tau$. Denote:

$$
S_{\text {rot }}(a, b)=\left(\begin{array}{cc}
\cos \int_{a}^{b} \Omega(\tau) d \tau & \sin \int_{a}^{b} \Omega(\tau) d \tau \\
-\sin \int_{a}^{b} \Omega(\tau) d \tau & \cos \int_{a}^{b} \Omega(\tau) d \tau
\end{array}\right)
$$

So the values of $y$ and $y^{\prime}$ at $\tau= \pm R$ are related by:

$$
\left(\begin{array}{c}
\sqrt{\Omega(R)} y(R) \\
\frac{1}{\sqrt{\Omega(R)}} y^{\prime}(R)
\end{array}\right) \approx S_{r o t}(-R, R) \cdot\left(\begin{array}{c}
\sqrt{\Omega(R)} y(-R) \\
\frac{1}{\sqrt{\Omega(R)}} y^{\prime}(-R)
\end{array}\right)
$$

(by time-reversibility, $\Omega(R)=\Omega(-R)$ ).

Let us now consider the behavior of solutions of (A.33) on the interval $\tau>R$. Here $\tau$ is large, and we estimate the solution of (A.34) as

$$
z(\tau)(1+\varepsilon z(\tau) / 2)=h \frac{1-M}{4} \tau^{2}\left(1+O\left(\tau^{-\alpha}\right)\right)+\sqrt{M h} \tau\left(1+O\left(\tau^{-\alpha}\right)\right) .
$$

Recall that we are interested only in the behavior for $|\tau| \leq \Delta t / \delta$, which corresponds to $z=o\left(\varepsilon^{-1}\right)$ (see (A.32) and (A.35)), so we may write

$$
z(\tau)=h \frac{1-M}{4} \tau^{2}\left(1+o(1)_{\rho \rightarrow 0}\right)+\sqrt{M h} \tau\left(1+o(1)_{\rho \rightarrow 0}\right)
$$


on the interval $\tau>R$. After scaling the time $\tau=\kappa \theta$ where $\kappa$ is given by (B.5), we find (after some algebraic manipulations) that equation (A.33) on this interval transforms into

$$
y^{\prime \prime}(\theta)+\frac{1+o(1)}{\left((1-P) \theta^{2}+P \theta(1+o(1))\right)^{\alpha+2}} y(\theta)=0,
$$

where $P(\rho, M)$ is given by (B.4). Since equation (B.24) limits to (B.8) as $\rho \rightarrow 0$, its scattering matrix is well approximated by the scattering matrix $\hat{S}$ of (B.8). Thus, returning to the time $\tau=\kappa \theta$, we obtain from (B.11),(B.9),(B.10) that

$$
\left(\begin{array}{c}
D_{0}^{+} \\
\kappa D_{1}^{+}
\end{array}\right)=\hat{S}\left(\begin{array}{c}
E_{1} \\
E_{2}
\end{array}\right) \approx \hat{S} \cdot S_{r o t}(\tau, \infty) \cdot\left(\begin{array}{c}
\sqrt{\kappa \Omega(\tau)} y(\tau) \\
\frac{1}{\sqrt{\kappa \Omega(\tau)}} \kappa y^{\prime}(\tau)
\end{array}\right)
$$

where $D_{0,1}^{+}$are the coefficients of the expansion (A.22) for the solutions of (A.33).

By time-reversibility, for the interval $\tau<-R$ we have

$$
\left(\begin{array}{c}
D_{0}^{-} \\
-\kappa D_{1}^{-}
\end{array}\right) \approx \sqrt{\kappa} \hat{S} \cdot S_{r o t}(-\infty, \tau) \cdot\left(\begin{array}{c}
\sqrt{\Omega(\tau)} y(\tau) \\
-\frac{1}{\sqrt{\Omega(\tau)}} y^{\prime}(\tau)
\end{array}\right)
$$

namely,

$$
\begin{aligned}
\left(\begin{array}{c}
\sqrt{\Omega(\tau)} y(\tau) \\
\frac{1}{\sqrt{\Omega(\tau)}} y^{\prime}(\tau)
\end{array}\right) & \approx \frac{1}{\sqrt{\kappa}}\left(\begin{array}{cc}
1 & 0 \\
0 & -1
\end{array}\right) \cdot S_{r o t}^{-1}(-\infty, \tau) \cdot \hat{S}^{-1}\left(\begin{array}{c}
D_{0}^{-} \\
-\kappa D_{1}^{-}
\end{array}\right) \\
& =\frac{1}{\sqrt{\kappa}} S_{r o t}(-\infty, \tau) \cdot\left(\begin{array}{cc}
1 & 0 \\
0 & -1
\end{array}\right) \cdot \hat{S}^{-1}\left(\begin{array}{c}
D_{0}^{-} \\
-\kappa D_{1}^{-}
\end{array}\right)
\end{aligned}
$$

From (B.23),(B.25) and (B.27) we find

$$
\begin{aligned}
\left(\begin{array}{c}
D_{0}^{+} \\
\kappa D_{1}^{+}
\end{array}\right) & \approx \hat{S} \cdot S_{\text {rot }}(R, \infty) \cdot S_{\text {rot }}(-R, R) \cdot S_{\text {rot }}(-\infty, R) \cdot\left(\begin{array}{cc}
1 & 0 \\
0 & -1
\end{array}\right) \cdot \hat{S}^{-1}\left(\begin{array}{c}
D_{0}^{-} \\
-\kappa D_{1}^{-}
\end{array}\right) \\
& =\hat{S}\left(\begin{array}{cc}
\cos \int_{-\infty}^{+\infty} \Omega(\tau) d \tau & \sin \int_{-\infty}^{+\infty} \Omega(\tau) d \tau \\
-\sin \int_{-\infty}^{+\infty} \Omega(\tau) d \tau & \cos \int_{-\infty}^{+\infty} \Omega(\tau) d \tau
\end{array}\right)\left(\begin{array}{cc}
1 & 0 \\
0 & -1
\end{array}\right) \hat{S}^{-1}\left(\begin{array}{cc}
1 & 0 \\
0 & -1
\end{array}\right)\left(\begin{array}{c}
D_{0}^{-} \\
\kappa D_{1}^{-}
\end{array}\right) .
\end{aligned}
$$

By (B.12), this gives us the following formula for the scattering matrix $S:\left(D_{0}^{-}, D_{1}^{-}\right) \rightarrow$ $\left(D_{0}^{+}, D_{1}^{+}\right)$:

$$
S \approx\left(\begin{array}{cc}
\sqrt{G g} \sin (\Psi+\varphi+\zeta) & \kappa g \sin (\Psi+2 \zeta) \\
\frac{G}{\kappa} \sin (\Psi+2 \varphi) & \sqrt{G g} \sin (\Psi+\varphi+\zeta)
\end{array}\right)
$$

where

$$
\Psi=\int_{-\infty}^{+\infty} \Omega(\tau) d \tau \approx \frac{2 J}{\rho} \sqrt{\frac{\alpha(\alpha+1)}{n-1}}
$$

and $G, g, \varphi, \zeta$ are the coefficients of the scattering matrix $\hat{S}$ that depend only on $P$ and $\alpha$ (see (B.17),(B.18),(B.2)). 
Now, like in the proof of Theorem 2, by virtue of (A.2),(A.25),(A.14),(A.16),(A.13),(3.7), (B.7),(B.28), we obtain the following formula ${ }^{13}$ for the trace of the monodromy matrix $A=B C$ :

$$
\operatorname{Tr}(A)=\frac{2 G}{\sqrt{h} \delta \kappa} d\left(1+\frac{d}{R}\right) \sin (\Psi+2 \varphi)(1+o(1))+2 \sqrt{G g} \sin (\Psi+\varphi+\zeta)\left(1+\frac{2 d}{R}\right)+o(1) .
$$

Equating $\operatorname{Tr}(A)$ to \pm 2 supply the stability intervals (B.14),(B.15); Since $\delta \kappa$ is small (see (B.7)) and $G$ is non-zero (by (B.13)), only the first term is of importance, and the stability intervals are created when $\Psi+2 \varphi \approx \pi k$, which gives (B.14) (see (B.29)). Formula (B.15) is found from:

$$
\Delta \rho\left|\frac{d \Psi}{d \rho}\right| \frac{2 G}{\sqrt{h} \delta \kappa} d\left(1+\frac{d}{R}\right) \approx 4
$$

\section{REFERENCES}

[1] M.-C. Arnaud, Difféomorphismes symplectiques de classe $c^{1}$ en dimension 4, C. R. Acad. Sci. Paris Ser. I Math. 331 (2000), no. 12, 1001-1004, In French.

[2] P. R. Baldwin, Soft billiard systems., Phys. D 29 (1988), no. 3, 321-342.

[3] P. Bálint and I. P. Tóth, Mixing and its rate in 'soft' and 'hard' billiards motivated by the Lorentz process, Phys. D 187 (2004), no. 1-4, 128-135, Microscopic chaos and transport in many-particle systems.

[4] __ Hyperbolicity in multi-dimensional Hamiltonian systems with applications to soft billiards, Discrete Contin. Dyn. Syst. 15 (2006), no. 1, 37-59.

[5] V.J. Donnay, Elliptic islands in generalized Sinai billiards, Ergod. Th. \& Dynam. Sys. 16 (1996), 975-1010.

[6] V.J. Donnay and C. Liverani, Potentials on the two-torus for which the Hamiltonian flow is ergodic., Commun. Math. Phys. 135 (1991), 267-302.

[7] A. Giorgilli, A. Delshams, E. Fontich, L. Galgani, and C. Simó, Effective stability for Hamiltonian systems near an elliptic point, with an application to the restricted three body problem, J. Diff. Eq. 77 (1989), no. 1, 167.

[8] S. V. Gonchenko, L. P. Shilnikov, and D. V. Turaev, Elliptic periodic orbits near a homoclinic tangency in four-dimensional symplectic maps and hamiltonian systems with three degrees of freedom, Regular and Chaotic Dynamics 3 (1998), no. 4, 3-26.

[9] _ Infinitely many elliptic periodic orbits in four dimensional symplectic diffeomorphism with a homoclinic tangency, Proc. Steklov Inst. Math. 244 (2004), 106-131.

[10] A. Kaplan, N. Friedman, M. Andersen, and N. Davidson, Observation of islands of stability in soft wall atom-optics billiards, Phys. Rev. Let. 87 (2001), no. 27, 274101-1-4.

[11] A. Krámli, N. Simányi, and D. Szász, Ergodic properties of semi-dispersing billiards. I. Two cylindric scatterers in the 3D torus, Nonlinearity 2 (1989), no. 2, 311-326.

[12] _ A "transversal" fundamental theorem for semi-dispersing billiards, Comm. Math. Phys. 129 (1990), no. 3, 535-560.

[13] _ The K-property of three billiard balls, Ann. of Math. (2) 133 (1991), no. 1, 37-72.

[14] The K-property of four billiard balls, Comm. Math. Phys. 144 (1992), no. 1, 107-148.

[15] N. S. Krylov, Works on the foundations of statistical physics, Princeton University Press, Princeton, N.J., 1979, Translated from the Russian by A. B. Migdal, Ya. G. Sinai and Yu. L. Zeeman, With a preface by A. S. Wightman, With a biography of Krylov by V. A. Fock, With an introductory article "The views of N. S. Krylov on the foundations of statistical physics" by Migdal and Fok, With a supplementary article "Development of Krylov's ideas" by Sinai, Princeton Series in Physics.

[16] I. Kubo, Perturbed billiard systems $i$ the ergodicity of the motion of a particle in a compound central field, Nagoya Math. J. 61 (1976), 1-57.

[17] J. E. Marsden, Generalized Hamiltonian mechanics: A mathematical exposition of non-smooth dynamical systems and classical Hamiltonian mechanics, Arch. Rational Mech. Anal. 28 (1967/1968), 323-361. MR MR0224935 (37 \#534)

\footnotetext{
${ }^{13}$ In particular, setting $\mu=0$ and $\alpha=1$ in (B.30) (so $M=P=0, \delta=\sqrt{\varepsilon / 2}, \rho=\sqrt{2 \varepsilon}, \kappa=$ $\left.\left(\frac{1}{\varepsilon} \frac{1}{n-1}\right)^{\frac{1}{4}}\left(\frac{4}{h}\right)^{\frac{3}{4}}, J(0)=\sqrt{h}\right)$ gives formula (4.1).
} 
[18] N.N. Nekhoroshev, An exponential estimate of the time of stability of near-integrable Hamiltonian systems, Russian Math. Surveys 32 (1977), no. 6, 1-65.

[19] S. Newhouse, Quasi-elliptic periodic points in conservative dynamical systems, Amer. J. Of Math. 99 (1977), no. 5, 1061-1087.

[20] A. Rapoport and V. Rom-Kedar, Nonergodicity of the motion in three-dimensional steep repelling dispersing potentials, Chaos 16 (2006), no. 4, 043108

[21] A. Rapoport, V. Rom-Kedar, and D Turaev, Approximating multi-dimensional Hamiltonian flows by billiards, to appear in CMP (2006).

[22] V. Rom-Kedar and D. Turaev, Big islands in dispersing billiard-like potentials, Physica D 130 (1999), 187210.

[23] ___ Big islands in dispersing billiard-like potentials, Physica D 130 (1999), no. 3,4, 187-210.

[24] R. Saghin and Z. Xia, Partial hyperbolicity of dense elliptic periodic points for $c^{1}$-generic symplectic diffeomorphisms, Trans. Amer. Math. Soc 358 (2006), 5119-5138.

[25] N. Simányi, The K-property of N billiard balls. I, Invent. Math. 108 (1992), no. 3, 521-548.

[26] _ The K-property of $N$ billiard balls. II. Computation of neutral linear spaces, Invent. Math. 110 (1992), no. 1, 151-172.

[27] _ Proof of the ergodic hypothesis for typical hard ball systems, Ann. Henri Poincaré 5 (2004), no. 2, 203-233

[28] N. Simányi, The Boltzmann-Sinai Ergodic Hypothesis in Full Generality (Without Exceptional Models), ArXiv Mathematics e-prints (2005)

[29] N. Simányi and D. Szász, Hard ball systems are completely hyperbolic, Ann. of Math. (2) 149 (1999), no. 1, 35-96.

[30] Ya.G. Sinai, On the foundations of the ergodic hypothesis for dynamical system of statistical mechanics, Dokl. Akad. Nauk. SSSR 153 (1963), 1261-1264.

[31] _ Dynamical systems with elastic reflections: Ergodic properties of scattering billiards, Russian Math. Sur. 25 (1970), no. 1, 137-189.

[32] Ya.G. Sinai and N.I. Chernov, Ergodic properties of some systems of two-dimensional disks and threedimensional balls, Uspekhi Mat. Nauk 42 (1987), no. 3(255), 153-174, 256, In Russian.

[33] F. Takens, Homoclinic points in conservative systems, Invent. Math. 18 (1972), no. 3-4, 267-292.

[34] D. Turaev and V. Rom-Kedar, Islands appearing in near-ergodic flows, Nonlinearity 11 (1998), no. 3, 575600 .

[35] D. Turaev and V. Rom-Kedar, Soft billiards with corners, J. Stat. Phys. 112 (2003), no. 3-4, 765-813.

[36] M. Wojtkowski, Principles for the design of billiards with nonvanishing lyapunov exponents, Comm. Math. Phys. 105 (1986), no. 3, 391-414.

ANNA.RAPOPORT@WEIZMANN.AC.IL, VERED.ROM-KEDAR@WEIZMANN.AC.IL, WEIZMANN INSTITUTE, REHOVOT, ISRAEL

TURAEV@CS.BGU.AC.IL, BEN GURION UNIVERSITY, BEER-SHEVA, ISRAEL. 